\title{
Slow and Steady, or Hard and Fast? A Systematic Review and Meta-Analysis of Studies Comparing Body Composition Changes between Interval Training and Moderate Intensity Continuous Training
}

\author{
James Steele $^{1}\left(\mathbb{D}\right.$, Daniel Plotkin ${ }^{2}$ D, Derrick Van Every ${ }^{2} \mathbb{D}$, Avery Rosa $^{2}$, Hugo Zambrano ${ }^{2}$, \\ Benjiman Mendelovits ${ }^{2}$, Mariella Carrasquillo-Mercado ${ }^{2}$, Jozo Grgic ${ }^{3}$ and Brad J. Schoenfeld ${ }^{2, *}$ \\ 1 School of Sport, Health and Social Sciences, Solent University, Southampton SO14 0YN, UK; \\ james.steele@solent.ac.uk \\ 2 Health Sciences Department, CUNY Lehman College, Bronx, NY 10468, USA; \\ danielplotkin96@gmail.com (D.P.); vaneverd@uwindsor.ca (D.V.E.); averyrosa1@gmail.com (A.R.); \\ hugo9523@gmail.com (H.Z.); benjimanbm1@gmail.com (B.M.); mariellacm123@gmail.com (M.C.-M.) \\ 3 Institute for Health and Sport, Victoria University, Melbourne, VIC 8001, Australia; jozo.grgic@live.vu.edu.au \\ * Correspondence: brad.schoenfeld@lehman.cuny.edu
}

check for updates

Citation: Steele, J.; Plotkin, D.; Van Every, D.; Rosa, A.; Zambrano, H.; Mendelovits, B.;

Carrasquillo-Mercado, M.; Grgic, J.; Schoenfeld, B.J. Slow and Steady, or Hard and Fast? A Systematic Review and Meta-Analysis of Studies Comparing Body Composition Changes between Interval Training and Moderate Intensity Continuous Training. Sports 2021, 9, 155. https:// doi.org/10.3390/sports9110155

Academic Editor: Gerald Mangine

Received: 26 October 2021

Accepted: 12 November 2021

Published: 18 November 2021

Publisher's Note: MDPI stays neutral with regard to jurisdictional claims in published maps and institutional affiliations.

Copyright: (c) 2021 by the authors. Licensee MDPI, Basel, Switzerland. This article is an open access article distributed under the terms and conditions of the Creative Commons Attribution (CC BY) license (https:/ / creativecommons.org/licenses/by/ $4.0 /)$.
Abstract: Purpose: To conduct a systematic review and multilevel meta-analysis of the current literature as to the effects of interval training (IT) vs moderate intensity continuous training (MICT) on measures of body composition, both on a whole-body and regional level. Methods: We searched English-language papers on PubMed/MEDLINE, Scopus, CINAHL, and sportrxiv for the following inclusion criteria: (a) randomized controlled trials that directly compared IT vs MICT body composition using a validated measure in healthy children and adults; (b) training was carried out a minimum of once per week for at least four weeks; (c) published in a peer-reviewed English language journal or on a pre-print server. Results: The main model for fat mass effects revealed a trivial standardized point estimate with high precision for the interval estimate, with moderate heterogeneity $\left(-0.016(95 \% \mathrm{CI}-0.07\right.$ to 0.04$\left.) ; I^{2}=36 \%\right)$. The main model for fat-free mass (FFM) effects revealed a trivial standardized point estimate with high precision for the interval estimate, with negligible heterogeneity $\left(-0.0004(95 \% \mathrm{CI}-0.05\right.$ to 0.05$\left.) ; I^{2}=16 \%\right)$. The GRADE summary of findings suggested high certainty for both main model effects. Conclusions: Our findings provide compelling evidence that the pattern of intensity of effort and volume during endurance exercise (i.e., IT vs MICT) has minimal influence on longitudinal changes in fat mass and FFM, which are likely to minimal anyway. Trial registration number: This study was preregistered on the Open Science Framework.

Keywords: intensity of effort; fat loss; fat mass; body fat; lean mass

\section{Introduction}

The relative components of fat mass and fat-free mass in the body, collectively termed body composition, has important implications for human health. Excessive levels of body fat show a high correlation with a panoply of disease states, including cardiovascular diseases, metabolic disorders, certain cancers, osteoarthritis, and respiratory conditions [1] Alternatively, low levels of fat-free mass are associated with a loss of strength, functional capacity, and reduced bone mineral density [2-4], impairing both the quality and quantity of life [1]. There is an interaction between these two components, whereby the combination of low levels of fat-free mass (FFM) and high levels of body fat potentiate each other, maximizing their impact on disability, morbidity, and mortality [5].

Exercise is commonly recommended as an intervention to improve body composition [6,7]. Interventional strategies often employed for this purpose include the following patterns. 
Moderate intensity continuous training (MICT), herein operationally defined as moderate intensity of effort exercise $(<80 \%$ peak heart rate or aerobic capacity) performed over a longer (relative to interval training bouts) single bout.

Interval training (IT), herein operationally defined as exercise performed in multiple shorter (relative to continuous training) bouts interspersed with recovery periods either at lower intensities of effort, or as complete rest.

IT is often subclassified into high intensity interval training (HIIT), herein operationally defined as high intensity of effort exercise (approximately $>80 \%$ peak heart rate or aerobic capacity) performed in multiple shorter bouts interspersed with recovery periods either at lower intensities of effort or as complete rest, and sprint interval training (SIT), herein operationally defined as maximal intensity of effort exercise ('all out' sprint) performed in multiple shorter bouts interspersed with recovery periods either at lower intensities of effort or as complete rest.

Although both MICT and IT show efficacy in improving body composition, controversy exists as to whether one strategy is superior to the other for this purpose. For example, an earlier meta-analysis by Keating et al. [8] reported little difference between MICT and IT for body fat reduction, highlighting that, over the short term, neither intervention produced clinically meaningful changes. Following this, Viana et al. [9] conducted a meta-analysis with results showing that IT produced a $28.5 \%$ greater reduction in fat mass than MICT. However, the paper was criticized for various methodological issues [10], ultimately leading to its retraction. More recently, Sultana et al. [11] carried out a meta-analysis that included a comparison of IT vs MICT. The analysis did not find a benefit to low-volume IT on measures of body composition when compared with MICT. However, they limited their analysis to only single measures per study of the constructs of interest (i.e., total body fat mass, body fat percentage, and lean body mass), whereas many studies often report several measures (e.g., regional measures). Furthermore, although several studies have also compared the effects of IT and MICT in younger populations, they limited the analysis to adults. Additionally, it is not clear from their analysis which pre-post test correlations were imputed and used for effect size calculations. The magnitude of pre-post test correlations used in calculations of pre-post control group design effect sizes using pooled baseline standard deviations can impact the heterogeneity determined in the meta-analysis [12]. Thus, although the standardized point estimates of Sultana et al. [11] models generally suggested little difference between conditions, the accompanying interval estimates for most outcomes included small effects in favor of either IT or MICT. Furthermore, their models had essentially no heterogeneity, although this may be the result of imputation of pre-post correlations that were relatively low. Application of multilevel meta-analytic models with robust variance estimation to handle multiple effects per study might yield a greater precision of estimates [13], and thus help to confirm whether small differences do in fact exist, and if so, in which direction. Additionally, extraction of information to permit calculation of pre-post test correlations within groups (i.e., see Higgins et al. [14]) would allow for a better estimate of the population pre-post test correlations and may reveal heterogeneity not identified in previous analyses. Lastly, although Sultana et al. [11] explored 'within-condition' effects for IT in studies that included a non-exercising control condition, they did not similarly explore this outcome for MICT training.

It also has been speculated that specific exercise-induced effects might occur for hypertrophy and regional fat mass. Endurance exercise may have beneficial effects on muscle hypertrophy, similar to that of resistance training [15], and some researchers highlight that IT, in particular, may produce a potent anabolic stimulus [16]. Furthermore, it has been suggested that IT may be more effective than MICT for abdominal fat mass reduction [17]. However, to our knowledge, no previous review has pooled data from research that directly compares changes in FFM between IT and MICT, nor specifically examined regional effects on changes in fat mass.

Lastly, although prior meta-analyses have considered between-conditions comparison of mean intervention effects [11], whether or not differences in the variance of treatment 
responses are present has been relatively less explored. A recent meta-analysis of aerobic exercise in overweight and obese children and adolescents found no evidence of 'true' interindividual response variation in fat loss [18]. However, numerous studies have purported that there may be inter-individual response variation to IT and MICT for a range of outcomes [19-21], and indeed it has been argued that such variation may mask differences between IT and MICT for fat loss [22]. Thus, we also sought to examine whether there is evidence of 'true' inter-individual response variation for body composition outcomes for both IT and MICT [23,24].

Given the gaps in the current literature, the purpose of this paper was to conduct a systematic review and multilevel meta-analysis of the current literature as to the effects of IT vs MICT on measures of body composition, both on a whole-body and regional level. Secondarily, we sought to determine if intensity of effort influences exercise adherence and/or adverse events, as well as whether inter-individual response to IT and MICT influences changes in body composition.

\section{Material and Methods}

This systematic review was conducted in accordance with the guidelines of the "Preferred Reporting Items for Systematic Reviews and Meta-Analyses" (PRISMA) [25]. The study was preregistered on the Open Science Framework (https:/ / osf.io/dq784), where the detailed prespecified methodological protocol can be viewed.

\subsection{Inclusion/Exclusion Criteria}

We included studies that met the following criteria: (a) randomized controlled trials (both within- and between-group designs) that directly compared IT vs MICT (both with and without adjuvant dietary interventions) for body composition using a validated measure (DXA, BodPod, hydrostatic weighing, BIA, skinfolds, ultrasound, magnetic resonance imaging, and computerized tomography) in healthy children and adults; (b) training was carried out a minimum of once per week for at least four weeks; (c) published in a peer-reviewed English language journal or on a pre-print server. We excluded studies that employed: (a) participants with co-morbidities that might impair aerobic capacity (respiratory conditions, musculoskeletal injury); and (b) an unbalanced resistance training component (e.g., one group performs resistance training whereas the other does not). Note, our original pre-registration failed to specify the particular intensity of effort and operationalization of this variable for determination of whether an IT intervention could be considered 'HIIT'. However, a small number of studies identified employed intensities of $>75 \%$ of peak heart rate or aerobic capacity for their IT conditions. Given our omission of specificity in pre-registration, we felt that these studies should be included, as there was still a reasonable difference in intensity of effort compared with the MICT conditions (typically $<60 \%$ ).

\subsection{Search Strategy}

We carried out a comprehensive search of the PubMed/MEDLINE, Scopus, CINAHL, and sportrxiv databases using the following Boolean string: (interval training OR intermittent training OR high intensity OR sprint interval training OR aerobic interval training OR HIIT OR HIIE OR high intensity interval training OR high-intensity interval training OR high intensity interval exercise OR high intensity intermittent exercise OR high-intensity intermittent exercise OR high intensity intermittent training OR high-intensity intermittent training) AND (continuous training OR moderate-intensity continuous exercise OR moderate intensity continuous exercise OR moderate-intensity continuous training OR moderate intensity continuous training OR endurance training) AND (body fat OR adiposity OR body composition OR abdominal fat OR visceral fat OR adipose tissue OR fat mass OR fat-free mass OR lean body mass OR lean mass OR muscle mass). Moreover, we screened the reference lists of articles retrieved to uncover any additional studies that might meet 
inclusion criteria, as described by Greenhalgh and Peacock [26]. The search was finalized on 6 March 2021; Figure 1 illustrates a flow chart of the search process.
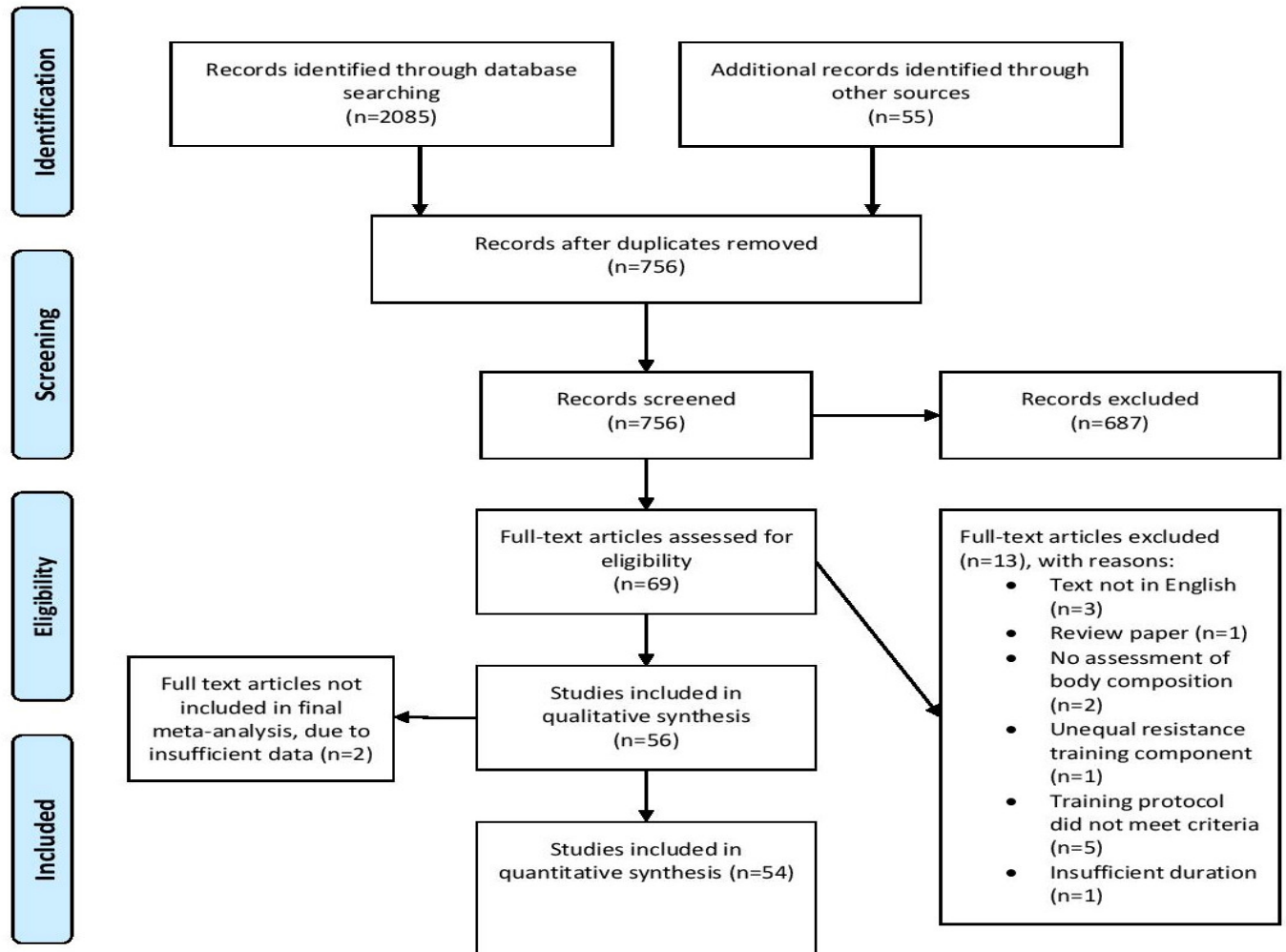

Figure 1. Flow chart of PRISMA search.

\subsection{Screening/Coding of Studies}

Search/screening was carried out separately by two researchers (DP and AR). These researchers read all titles and abstracts and then reviewed full texts for papers deemed relevant based on their title and abstract. Decisions then were made as to whether a study warranted inclusion based on the stated criteria. Any disputes on the inclusion of a given study were settled by a third researcher (MCM).

After determining which studies met inclusion, two researchers (DV and HZ) separately coded the following variables for each study: authors, title and year of publication, sample size, sex, body mass index (BMI), training status, age, description of the training intervention (duration, intensity, frequency, modality), work matched (yes/no), nutrition controlled (yes/no), method for body comp assessment (e.g., DXA, BodPod, BIA, hydrostatic weighing, skinfolds, MRI, CT, ultrasound), number of adverse effects associated with the training intervention, adherence to the given training program, mean preand post-study body composition values in addition to pre-post change scores with the corresponding standard deviation or standard error, and where change score standard deviations were not reported we extracted information to allow their calculation, including confidence intervals for change scores or within-group pre-post $t$ statistics or $p$ values (where $p$ values were reported only to the studies' level of alpha (e.g., $p<0.05$ ) we took this as a conservative value). In cases where body composition data were not reported numerically, we either extracted the data from graphs when available via online software, or attempted to contact the study's authors. Coding was cross-checked between reviewers, with any discrepancies resolved by mutual consensus. Consistent with the guidelines of Cooper et al. [27], 30\% of the included studies were randomly selected for re-coding to assess for potential coder drift by a third researcher (BM). Agreement was calculated by dividing the number of variables coded the same by the researchers by the total number of variables; acceptance required a mean agreement of 0.90 to avoid re-extraction entirely, and 
after this was met, only those with differing codes were checked and updated. Extracted data was also double-checked after this process by the lead author (JS) prior to analysis.

\subsection{Methodological Quality and Certainty of Evidence}

Two of the authors independently evaluated each study (JG and BJS) using the 11-point Physiotherapy Evidence Database (PEDro) scale, which has been validated to assess the methodologic quality of randomized trials [28] with acceptable inter-rater reliability [29]. Any discrepancies in agreement on a given scale item were settled by mutual agreement between the researchers. Given that it is infeasible to blind participants and investigators in supervised exercise interventions, we opted to remove assessment items specific to blinding (numbers 5, 6, and 7 in the scale). After eliminating these items, this created a modified 8-point PEDro scale with a maximum value of 7 (the first item is excluded from the total score). The qualitative methodological ratings were amended, similar to those used in previous exercise-related systematic reviews [30], as follows: "excellent" (6-7 points); "good" (5 points); "moderate" (4 points); and "poor" (0-3 points). We also followed the Grading of Recommendations, Assessment, Development and Evaluations (GRADE) framework [31] for evaluating the certainty of evidence with respect to our primary pre-registered outcomes (absolute fat mass, and absolute lean/fat free mass). We used the GRADEpro online tool [32] for this assessment and generation of the summary of findings table. It should be noted though that we did not pre-register the use of the GRADE approach to evaluate the evidence presented but decided a posteriori that the assessment would enhance the ability to draw practical inferences from the data.

\subsection{Statistical Analyses}

Quantitative synthesis of data was performed with the 'metafor' [33] package in $\mathrm{R}$ (v 4.0.2; R Core Team, https: / / www.r-project.org/). All analysis code and data are openly available in the Supplementary Materials (https:/ / osf.io/6karz/). Studies were grouped by design (i.e., within- or between-group), and depending on reporting in individual studies, either post or delta comparisons, or pre-post comparison designs [12] for the purposes of appropriate calculation of standardized effects (Hedges' g) using the escalc function in metafor were carried out. We used the pooled group baseline standard deviation as the numerator as per Morris (29). Standardized effect sizes were interpreted as per Cohen's [34] thresholds: trivial $(<0.2)$, small $(0.2$ to $<0.5)$, moderate $(0.5$ to $<0.8)$, and large $(\geq 0.8)$. Standardized effects were calculated in such a manner that a positive effect size value favored the IT conditions.

As there was a nested structure to the effect sizes calculated from the studies included (i.e., multiple effects nested within groups and nested within studies), multilevel mixed effect meta-analyses with both study and intra-study groups included as random effects in the model were performed. Cluster robust point estimates and the precision of those estimates using 95\% compatibility (confidence) intervals (CIs) were produced, weighted by the inverse sampling variance to account for the within- and between-study variance $\left(\tau^{2}\right)$. Restricted maximal likelihood estimation was used in all models. Two main models were produced for both pre-registered main outcomes (absolute fat mass and FFM), including all standardized effect sizes, to provide a general estimate of the comparative treatment effects. All other models were considered secondary and exploratory analyses.

For all models, we avoided dichotomizing the existence of an effect for the main results and therefore did not employ traditional null hypothesis significance testing, which has been extensively critiqued $[35,36]$. Instead, we considered the implications of all results compatible with these data, from the lower limit to the upper limit of the interval estimates, with the greatest interpretive emphasis placed on the point estimate. Given the large number of included studies and effects, the main models are visualized here using ordered caterpillar plots to aid interpretation, as opposed to traditional forest plots containing study characteristics. Note that all study characteristics are available in the data file in the 
Supplementary Materials (https:/ / osf.io/dumq8/), as are more traditional forest plots for the main models (see folder "Outputs and Figures" at https:/ / osf.io/6karz/).

The risk of small study bias was examined visually through contour-enhanced funnel plots. $Q$ and $I^{2}$ statistics were also produced and reported [37]. A significant $Q$ statistic is typically considered indicative of effects likely not being drawn from a common population. $I^{2}$ values indicate the relative degree of heterogeneity in the effects that are not due to sampling variance and are qualitatively interpreted as: $0-40 \%$ : not important, $30-60 \%$ : moderate heterogeneity, $50-90 \%$ : substantial heterogeneity, and $75-100 \%$ : considerable heterogeneity [38]. For within-participant effects, pre-post correlations for measures are often not reported in original studies; thus, for those studies where we had standard deviations for pre-, post-, and change scores (or were able to calculate the latter from confidence intervals, $\mathrm{t}$ statistics, or $p$ values) we calculated the pre-post correlations directly as:

$$
r_{\text {pre-post }}=\frac{S D_{\text {pre }}^{2}+S D_{\text {post }}^{2}+S D_{\text {change }}^{2}}{2 \times S D_{\text {pre }} \times S D_{\text {post }}}
$$

and imputed the median correlation coefficient to studies as an appropriate estimate of the population parameter.

In addition to the main models, we secondarily produced models for relative fat and FFM (i.e., as a percentage of body mass), and refit all models using delta scores (i.e., changes) of outcomes in the raw units of measurement (i.e., kilograms and percentages) to facilitate interpretation in a complementary fashion. We also produced models where studies included a non-training control arm that examined the between-condition treatment effects for both IT vs CON, and MICT vs CON, to determine the 'within-condition' effect estimates on both their standardized and raw scales, i.e., the true treatment effect of performing IT or MICT alone.

We planned to conduct exploratory subgroup and moderation analyses across standardized effects for the following: work matched/unmatched, modality of training (ambulatory, cycling, or other), sex (proportion of sample as males), age (years), BMI ( $\left.\mathrm{kg} \cdot \mathrm{m}^{2}\right)$, intervention characteristics including level of intensity of effort for IT (i.e., SIT vs HIIT), withinsession IT interval number and duration and their interaction, duration of MICT sessions, the difference (i.e., MICT minus IT) in total weekly exercise duration (frequency $\times$ duration), and duration of interventions (weeks), method of body composition measurement (DXA, BIA, skinfolds, etc.), body composition region of measurement (upper, lower, trunk), and whether nutrition was controlled or uncontrolled. Note, we originally mentioned exploration of moderators for both standardised and unstandardised effects in our preregistration. However, we ultimately opted to just explore standardised effects for absolute fat mass and FFM outcomes to compliment and explore heterogeneity in our main models. Furthermore, we adapted the operationalization of some moderators (e.g., intervention characteristics such as total weekly exercise duration) and some we could not explore fully given the number of effects available for certain sub-groups (these are noted in the analysis code). We also fit further (not pre-registered) models to examine adherence (number of attended sessions as a proportion of number of prescribed sessions) and dropout (number of participants dropped out as a proportion of number of participants randomized) proportions, as well as a Poisson regression model for adverse event count data (per 1000 person-sessions). All exploratory models utilized the same multilevel mixed-effects structure and specifications as the main models.

As a final exploratory (not pre-registered) analysis, we examined the variation in responses between both IT and MICT conditions. We sought to identify whether there was evidence of 'true' inter-individual variation from within-participant variability and/or participant-by-treatment interaction in responses to interventions by comparing the standard deviations for change scores with those of non-exercise control conditions [23,39]. We identified a mean-variance (on both the raw and log-transformed scales) relationship across studies for change scores (see https:/ / osf.io/6zb8y/). Thus, we opted to adjust for this by employing a multilevel meta-regression of the log-transformed change score standard 
deviations, adjusted for the log-change score mean [40], calculated such that positive values showed that intervention condition (i.e., IT and MICT) variation exceeded control condition variation - thus suggesting evidence of 'true' inter-individual response variation. Where studies did not report change score standard deviations, or we were unable to calculate it directly, this was estimated using the imputed median pre-post correlation coefficient noted above as:

$$
S D_{\text {change }}=\sqrt{S D_{\text {pre }}^{2}+S D_{\text {post }}^{2}-\left(2 \times r_{\text {pre-post }} \times S D_{\text {pre }} \times S D_{\text {post }}\right)}
$$

Note that, given the different measurement devices used in individual studies, we accepted pragmatically the inherent assumptions built into this comparison of a constant Gaussian measurement error (i.e., that measurement error does not scale in a non-linear fashion with measured scores).

\section{Results}

\subsection{Search Results}

From the initially reviewed 2085 search results, a total of 56 studies were determined as meeting the inclusion criteria for our analysis. Two studies stated that body composition measures were performed, but did not report information on this outcome in the manuscript [41,42]. Attempts to obtain the data from the corresponding authors proved unsuccessful. Thus, we analyzed 54 studies that compared the effects of IT and MICT on measures of body composition. Table 1 presents a summary of the methods of the included studies. Table 2 presents descriptive information as to the included studies. Figure 2 shows the contour enhanced funnel plot for all effects from these studies. Inspection of the funnel plot did not reveal any obvious small study bias.

Table 1. Methods of included studies.

\begin{tabular}{|c|c|c|c|c|c|c|c|}
\hline Study & $\begin{array}{c}\text { Sample } \\
\text { Population } \\
\text { (age) }\end{array}$ & $\begin{array}{l}\text { Duration } \\
\text { (weeks) }\end{array}$ & Group (n) & Modality/Intensity & $\begin{array}{l}\text { Frequency } \\
\text { (weeks) }\end{array}$ & $\begin{array}{l}\text { Time per } \\
\text { Session }\end{array}$ & $\begin{array}{c}\text { Body } \\
\text { Composition } \\
\text { Method }\end{array}$ \\
\hline [43] & $\begin{array}{l}\text { Obese } \\
\text { children } \\
\text { (aged 8-12 } \\
\text { years) }\end{array}$ & 12 & $\begin{array}{l}\text { MICT: } 15 \\
\text { IT: } 15\end{array}$ & $\begin{array}{l}\text { MICT: } 80 \% \text { of peak heart } \\
\text { rate } \\
\text { IT: } 3-6 \text { sets of } 60 \text { s sprint at } \\
100 \% \text { of the peak velocity } \\
\text { with } 3 \text { min active recovery } \\
\text { period at } 50 \% \text { of the } \\
\text { exercise velocity. }\end{array}$ & $\begin{array}{l}\text { MICT: } 2 \times \\
\text { IT: } 2 \times\end{array}$ & $\begin{array}{l}\text { MICT: } 30-60 \\
\text { min } \\
\text { IT: } 9-18 \mathrm{~min}\end{array}$ & BIA \\
\hline [44] & $\begin{array}{l}\text { Young adults } \\
\text { with } \\
\text { intellectual } \\
\text { disabilities } \\
\text { (aged 16-18 } \\
\text { years) }\end{array}$ & 15 & $\begin{array}{l}\text { MICT: } 15 \\
\text { IT: } 17 \\
\text { CON: } 14\end{array}$ & $\begin{array}{c}\text { MICT: Cycling, } \\
\text { walking/running, } \\
\text { stepping at } 30 \% \text { peak } \\
\text { watts } \\
\text { IT: First } 7 \text { weeks: } 10,15 \mathrm{~s} \\
\text { sprint bouts at ventilatory } \\
\text { threshold (100 + RPM), } 45 \\
\text { s recovery period at } 50 \\
\text { RPM } \\
\text { Weeks } 8-15: \text { intensity } \\
\text { increased to } 110 \% \text { VT }\end{array}$ & $\begin{array}{l}\text { MICT: } 2 \times \\
\text { IT: } 2 \times\end{array}$ & $\begin{array}{l}\text { MICT: } 40 \mathrm{~min} \\
\text { IT: } 40 \mathrm{~min}\end{array}$ & BIA \\
\hline [45] & $\begin{array}{c}\text { Down } \\
\text { syndrome } \\
\text { adults (mean } \\
\text { age } 34 \text { years) }\end{array}$ & 12 & $\begin{array}{l}\text { MICT: } 13 \\
\text { IT: } 13 \\
\text { CON: } 16\end{array}$ & $\begin{array}{c}\text { MICT: Continuous } \\
\text { cycling/walking at } \\
\text { 70-80\% } \mathrm{VO}_{2} \text { peak, } 85 \% \\
\text { after week } 6 \\
\text { IT: } 10 \times 30 \text { s sprints, } 90 \mathrm{~s} \\
\text { rest period }\end{array}$ & $\begin{array}{l}\text { MICT: } 3 \times \\
\text { IT: } 3 \times\end{array}$ & $\begin{array}{l}\text { MICT: } 30 \mathrm{~min} \\
\text { IT: } 30 \mathrm{~min}\end{array}$ & BIA \\
\hline
\end{tabular}


Table 1. Cont.

\begin{tabular}{|c|c|c|c|c|c|c|c|}
\hline Study & $\begin{array}{c}\text { Sample } \\
\text { Population } \\
\text { (age) }\end{array}$ & $\begin{array}{c}\text { Duration } \\
\text { (weeks) }\end{array}$ & Group (n) & Modality/Intensity & $\begin{array}{l}\text { Frequency } \\
\text { (weeks) }\end{array}$ & $\begin{array}{l}\text { Time per } \\
\text { Session }\end{array}$ & $\begin{array}{c}\text { Body } \\
\text { Composition } \\
\text { Method }\end{array}$ \\
\hline [46] & $\begin{array}{c}\text { Adolescents } \\
\text { (mean age } 16 \\
\text { years) }\end{array}$ & 7 & $\begin{array}{l}\text { MICT: } 16 \\
\text { IT: } 17 \\
\text { CON: } 24\end{array}$ & $\begin{array}{c}\text { MICT: Running at } \\
\text { moderate intensity at } 70 \% \\
\mathrm{VO}_{2} \max \left(\mathrm{VO}_{2} \max \right. \\
\text { retested at week } 4) \\
\text { IT: } 4-6 \text { all-out sprints } \times \\
30 \mathrm{~s}, 30 \text { s recovery period } \\
\text { (20 s recovery period for } \\
\text { week } 7)\end{array}$ & $\begin{array}{l}\text { MICT: } 3 \times \\
\text { IT: } 3 \times\end{array}$ & $\begin{array}{l}\text { MICT: } 20 \mathrm{~min} \\
\text { IT: } 16 \mathrm{~min}\end{array}$ & Skinfolds \\
\hline [47] & $\begin{array}{c}\text { Children } \\
\text { (mean age: } 11 \\
\text { years) }\end{array}$ & 8 & $\begin{array}{l}\text { MICT: } 16 \\
\text { IT: } 18\end{array}$ & $\begin{array}{l}\text { MICT: Running at } 65-75 \% \\
\quad \text { HRmax } \\
\text { IT: } 3-6 \text { bouts, } 20 \mathrm{~s} \\
\text { max-effort sprint, } 60 \mathrm{~s} \text { rest } \\
\text { period (40 s at week } 5,20 \mathrm{~s} \\
\quad \text { at week } 8 \text { ) }\end{array}$ & $\begin{array}{l}\text { MICT: } 3 \times \\
\text { IT: } 3 \times\end{array}$ & $\begin{array}{c}\text { MICT: } \\
\text { 4-9 min } \\
\text { IT: } 4-9 \text { min }\end{array}$ & BIA \\
\hline [48] & $\begin{array}{l}\text { Obese adults } \\
\text { (mean age: } \\
39 \text { years) }\end{array}$ & 12 & $\begin{array}{l}\text { MICT: } 6 \\
\text { IT: } 6\end{array}$ & $\begin{array}{l}\text { MICT: Brisk walking at } 4 \\
\text { METs } \\
\text { IT: } 4-7 \text { intervals at a 2:1 } \\
\text { ratio, then } 5 \text { boxing drills } \\
\times 3 \text { intervals at a } 2: 1 \text { ratio, } \\
\text { RPE } 15-17 \text { (>75\% HRmax) }\end{array}$ & $\begin{array}{l}\text { MICT: } 4 \times \\
\text { IT: } 4 \times\end{array}$ & $\begin{array}{l}\text { MICT: } 50 \mathrm{~min} \\
\text { IT: } 50 \mathrm{~min}\end{array}$ & Skinfolds \\
\hline [49] & $\begin{array}{c}\text { Obese adult } \\
\text { men } \\
\text { (mean age: } \\
25 \text { years) }\end{array}$ & 4 & $\begin{array}{l}\text { MICT: } 8 \\
\text { IT: } 8\end{array}$ & $\begin{array}{c}\text { MICT: Continuous cycling } \\
@ 65 \% \mathrm{VO}_{2} \text { peak } \\
\text { IT: } 4-7 \text { sprints } \times 30 \mathrm{~s} \text { at } \\
200 \% \mathrm{~W} \text {-max, } 120 \mathrm{~s} \text { at } 30 \\
\text { W in-between }\end{array}$ & $\begin{array}{l}\text { MICT: } 5 \times \\
\text { IT: } 3 \times\end{array}$ & $\begin{array}{c}\text { MICT: } \\
\text { 40-60 min } \\
\text { IT: } \\
\text { 10-17.5 min }\end{array}$ & DXA \\
\hline$[50]$ & $\begin{array}{l}\text { Colorectal } \\
\text { cancer } \\
\text { survivors } \\
\text { (mean age: } \\
62 \text { years) }\end{array}$ & 4 & $\begin{array}{l}\text { MICT: } 14 \\
\text { IT: } 21\end{array}$ & $\begin{array}{c}\text { MICT: Continuous cycling } \\
\text { at } 50-70 \% \text { HRpeak } \\
\text { IT: } 4 \text { cycling intervals } \times \\
240 \text { s at } 85-95 \% \text { HRpeak, } \\
180 \text { s active rest }\end{array}$ & $\begin{array}{l}\text { MICT: } 3 \times \\
\text { IT: } 3 \times\end{array}$ & $\begin{array}{l}\text { MICT: } 50 \mathrm{~min} \\
\text { IT: } 38 \mathrm{~min}\end{array}$ & DXA \\
\hline [51] & $\begin{array}{l}\text { Obese } \\
\text { children } \\
\text { (aged 7-16 } \\
\text { years) }\end{array}$ & 12 & $\begin{array}{l}\text { MICT: } 22 \\
\text { IT: } 18 \\
\text { CON: } 16\end{array}$ & $\begin{array}{l}\text { MICT: } 60-70 \% \text { HRmax } \\
\text { IT: } 4 \text { intervals of } 240 \mathrm{~s} \text { at } \\
85-95 \% \text { HRmax, } 180 \mathrm{~s} \\
\text { active recovery at } 50-70 \% \\
\text { HRmax }\end{array}$ & $\begin{array}{l}\text { MICT: } 3 \times \\
\text { IT: } 3 \times\end{array}$ & $\begin{array}{l}\text { MICT: } 44 \mathrm{~min} \\
\text { IT: } 28 \mathrm{~min}\end{array}$ & $\begin{array}{l}\text { DXA and } \\
\text { ADP }\end{array}$ \\
\hline [52] & $\begin{array}{l}\text { Adult men at } \\
\text { risk for } \\
\text { insulin } \\
\text { resistance } \\
\text { (mean age: } \\
48 \text { years) }\end{array}$ & 12 & $\begin{array}{l}\text { MICT: } 16 \\
\text { IT: } 21\end{array}$ & $\begin{array}{c}\text { MICT: Worked towards } 6 \\
\text { kcal/kg per week for } 6 \\
\text { weeks (+2 per week until } \\
12 \mathrm{kcal} / \mathrm{kg} \text { per week) } \\
\text { treadmill at } 50-70 \% \mathrm{VO}_{2} \\
\text { max } \\
\text { IT: Performed MICT } \\
\text { protocol until week } 6 \text { then } \\
\text { transitioned, } 2-8 \text { bouts of } \\
60 \mathrm{~s} \text { at } 90-95 \% \mathrm{VO}_{2} \text { max } \\
60 \mathrm{~s} \text { recovery period at } \\
50 \% \mathrm{VO}_{2} \text { max }\end{array}$ & $\begin{array}{c}\text { MICT: } 3-4 \times \\
\text { IT: } 3-4 \times\end{array}$ & $\begin{array}{l}\text { MICT: Work- } \\
\text { dependent } \\
\text { IT: } 4-16 \mathrm{~min}\end{array}$ & DXA \\
\hline [53] & $\begin{array}{l}\text { Obese young } \\
\text { women } \\
\text { (mean age: } \\
22 \text { years) }\end{array}$ & 8 & $\begin{array}{l}\text { MICT: } 7 \\
\text { IT: } 7 \\
\text { CON: } 6\end{array}$ & $\begin{array}{c}\text { MICT: Continuous } \\
\text { treadmill at } 50-70 \% \text { at } \\
\text { HRpeak } \\
\text { IT: } 4 \text { intervals of } 240 \mathrm{~s} \text { at } \\
\text { 85-95\% HRpeak, } 180 \mathrm{~s} \\
\text { active rest }\end{array}$ & $\begin{array}{l}\text { MICT: } 3 \times \\
\text { IT: } 3 \times\end{array}$ & $\begin{array}{l}\text { MICT: } 41 \mathrm{~min} \\
\text { IT: } 33 \mathrm{~min}\end{array}$ & BIA \\
\hline
\end{tabular}


Table 1. Cont.

\begin{tabular}{|c|c|c|c|c|c|c|c|}
\hline Study & $\begin{array}{c}\text { Sample } \\
\text { Population } \\
\text { (age) }\end{array}$ & $\begin{array}{l}\text { Duration } \\
\text { (weeks) }\end{array}$ & Group (n) & Modality/Intensity & $\begin{array}{l}\text { Frequency } \\
\text { (weeks) }\end{array}$ & $\begin{array}{l}\text { Time per } \\
\text { Session }\end{array}$ & $\begin{array}{c}\text { Body } \\
\text { Composition } \\
\text { Method }\end{array}$ \\
\hline [54] & $\begin{array}{c}\text { Sedentary } \\
\text { young men } \\
\text { (mean age: } 21 \\
\text { years) }\end{array}$ & 8 & $\begin{array}{l}\text { MICT: } 6 \\
\text { IT: } 6\end{array}$ & $\begin{array}{l}\text { MICT: Continuous } \\
\text { treadmill at } 70-80 \% \mathrm{VO}_{2} \\
\quad \text { max } \\
\text { IT: } 12 \text { intervals of } 60 \mathrm{~s} \text { at } \\
90-110 \% \mathrm{VO}_{2} \text { max, } 60 \mathrm{~s} \\
\text { rest period }\end{array}$ & $\begin{array}{l}\text { MICT: } 3 \times \\
\text { IT: } 3 \times\end{array}$ & $\begin{array}{l}\text { MICT: } 30 \mathrm{~min} \\
\text { IT: } 30 \mathrm{~min}\end{array}$ & DXA \\
\hline [55] & $\begin{array}{l}\text { Obese young } \\
\text { men (aged } \\
\text { 17-22 years) }\end{array}$ & 6 & $\begin{array}{l}\text { MICT: } 13 \\
\text { IT: } 15\end{array}$ & $\begin{array}{l}\text { MICT: Cycling at } 55-65 \% \\
\text { VO }_{2} \text { peak } \\
\text { IT: } 4 \text { intervals of } 240 \mathrm{~s} \text { at } \\
15 \% \text { APmax, then } 30 \mathrm{~s} \text { at } \\
85 \% \text { APmax, then } 120 \mathrm{~s} \text { at } \\
15 \% \text { APmax }\end{array}$ & $\begin{array}{l}\text { MICT: } 5 \times \\
\text { IT: } 3 \times\end{array}$ & $\begin{array}{l}\text { MICT: } 45-60 \\
\text { min } \\
\text { IT: } 20 \min \end{array}$ & DXA \\
\hline [56] & $\begin{array}{l}\text { Overweight } \\
\text { men (aged } \\
\text { 20-40 years) }\end{array}$ & 12 & $\begin{array}{l}\text { MICT: } 12 \\
\text { IT: } 10\end{array}$ & $\begin{array}{l}\text { MICT: Walking/jogging at } \\
65 \% \text { HRmax } \\
\text { IT: 6-12 intervals of } 60 \mathrm{~s} \text { at } \\
90-95 \% \text { HRmax, } 60 \mathrm{~s} \\
\text { active rest }\end{array}$ & $\begin{array}{l}\text { MICT: } 3 \times \\
\text { IT: } 3 \times\end{array}$ & $\begin{array}{l}\text { MICT: } 18-35 \\
\text { min } \\
\text { IT: } 12-24 \text { min }\end{array}$ & DXA \\
\hline [57] & $\begin{array}{c}\text { Sedentary } \\
\text { young men } \\
\text { (mean age: } 27 \\
\text { years) }\end{array}$ & 12 & $\begin{array}{l}\text { MICT: } 10 \\
\text { IT: } 9 \\
\text { CON: } 6\end{array}$ & $\begin{array}{c}\text { MICT: Continuous cycling } \\
\text { at } 70 \% \text { HRmax } \\
\text { IT: } 3 \text { all-out intervals of } 20 \\
\text { s at } 0.5 \mathrm{~kg} / \mathrm{kg} \text { resistance, } \\
120 \mathrm{~s} \text { low-intensity active } \\
\text { rest }\end{array}$ & $\begin{array}{l}\text { MICT: } 3 \times \\
\text { IT: } 3 \times\end{array}$ & $\begin{array}{l}\text { MICT: } 45 \mathrm{~min} \\
\text { IT: } 10 \mathrm{~min}\end{array}$ & $\mathrm{ADP}$ \\
\hline [58] & $\begin{array}{l}\text { Male police } \\
\text { officers } \\
\text { (mean age: } \\
39 \text { years) }\end{array}$ & 8 & $\begin{array}{l}\text { MICT: } 11 \\
\text { IT: } 11\end{array}$ & $\begin{array}{c}\text { MICT: Continuous } \\
\text { running at } 60-75 \% \\
\text { V-shuttle max } \\
\text { IT: 7-10 intervals of } \\
\text { 85-100\% V-shuttle max } \\
\text { (V-shuttle based on } \\
\text { individual shuttle test } \\
\text { results) }\end{array}$ & $\begin{array}{l}\text { MICT: } 3 \times \\
\text { IT: } 3 \times\end{array}$ & $\begin{array}{l}\text { MICT: } \\
\text { 27.8-33.4 } \min \\
\text { IT: } 14.8-19.1 \\
\text { min }\end{array}$ & DXA \\
\hline [59] & $\begin{array}{l}\text { Overweight } \\
\text { young } \\
\text { women } \\
\text { (mean age: } \\
20 \text { years) }\end{array}$ & 6 & $\begin{array}{l}\text { MICT: } 29 \\
\text { IT: } 23\end{array}$ & $\begin{array}{l}\text { MICT: Continuous cycling } \\
\text { at } 60-70 \% \text { HRR } \\
\text { IT: } 5-7 \text { all-out intervals of } \\
30 \mathrm{~s}, 240 \mathrm{~s} \text { active recovery }\end{array}$ & $\begin{array}{l}\text { MICT: } 3 \times \\
\text { IT: } 3 \times\end{array}$ & $\begin{array}{l}\text { MICT: } 20-30 \\
\min \\
\text { IT: } 22.5-31.5 \\
\min \end{array}$ & DXA \\
\hline$[60]$ & $\begin{array}{l}\text { Healthy, } \\
\text { sedentary } \\
\text { older adults } \\
\text { (aged } \\
\text { 55-79 years) }\end{array}$ & 8 & $\begin{array}{l}\text { MICT: } 14 \\
\text { IT: } 15 \\
\text { CONT: } 14\end{array}$ & $\begin{array}{c}\text { MICT: } 70 \% \text { of peak heart } \\
\text { rate } \\
\text { IT: } 4 \times 4 \text { min intervals at } \\
90 \% \text { of peak heart rate } \\
\text { with } 3 \times 3 \text { min active } \\
\text { recovery periods at } 70 \% \text { of } \\
\text { peak heart rate. }\end{array}$ & $\begin{array}{l}\text { MICT: } 4 \times \\
\text { IT: } 4 \times\end{array}$ & $\begin{array}{l}\text { MICT: } 47 \mathrm{~min} \\
\text { IT: } 40 \mathrm{~min}\end{array}$ & DXA \\
\hline [61] & $\begin{array}{c}\text { Inactive, } \\
\text { overweight } \\
\text { adults (aged } \\
\text { 18-55 years) }\end{array}$ & 12 & $\begin{array}{l}\text { MICT: } 11 \\
\text { IT: } 11 \\
\text { CONT: } 11\end{array}$ & $\begin{array}{l}\text { MICT: } 50-65 \% \mathrm{VO}_{2} \text { peak } \\
\text { IT: cycling, } 4-6 \text { sets of } \\
30-60 \text { s at } 120 \% \mathrm{VO}_{2} \text { peak } \\
\text { with } 120-180 \mathrm{~s} \text { at } 30 \mathrm{~W} \text {. }\end{array}$ & $\begin{array}{l}\text { MICT: } 3 \times \\
\text { IT: } 3 \times\end{array}$ & $\begin{array}{c}\text { MICT: } \\
\text { 30-45 min } \\
\text { IT: } 20-24 \mathrm{~min}\end{array}$ & DXA \\
\hline [62] & $\begin{array}{c}\text { Obese } \\
\text { adolescents } \\
\text { (mean age: } \\
13 \text { years) }\end{array}$ & 12 & $\begin{array}{l}\text { MICT: } 15 \\
\text { IT: } 14\end{array}$ & $\begin{array}{l}\text { MICT: } 60-70 \% \text { of } \mathrm{VO}_{2} \max \\
\text { IT: running for } 2 \text { min at } \\
80-90 \% \text { of } \mathrm{VO}_{2} \max \\
\text { followed by recovery } \\
\text { periods of } 1 \text { min. }\end{array}$ & $\begin{array}{l}\text { MICT: } 3 \times \\
\text { IT: } 3 \times\end{array}$ & $\begin{array}{l}\text { MICT: } \\
\text { 30-40 min }\end{array}$ & Skinfolds \\
\hline
\end{tabular}


Table 1. Cont.

\begin{tabular}{|c|c|c|c|c|c|c|c|}
\hline Study & $\begin{array}{c}\text { Sample } \\
\text { Population } \\
\text { (age) }\end{array}$ & $\begin{array}{l}\text { Duration } \\
\text { (weeks) }\end{array}$ & Group (n) & Modality/Intensity & $\begin{array}{l}\text { Frequency } \\
\text { (weeks) }\end{array}$ & $\begin{array}{l}\text { Time per } \\
\text { Session }\end{array}$ & $\begin{array}{c}\text { Body } \\
\text { Composition } \\
\text { Method }\end{array}$ \\
\hline [63] & $\begin{array}{l}\text { Overweight, } \\
\text { inactive } \\
\text { adults (aged } \\
\text { 35-60 years) }\end{array}$ & 12 & $\begin{array}{c}\text { MICT: } 17 \\
\text { IT (AIT): } 11 \\
\text { IT (MVIT): } \\
16\end{array}$ & $\begin{array}{l}\text { MICT: Walking, } 65-75 \% \text { of } \\
\text { HRmax } \\
\text { IT (AIT): jogging, } 4 \text { cycles } \\
\text { of } 4 \text { min at } 85-95 \% \\
\text { HRmax followed by } 3 \text { min } \\
\text { recovery at } 65-75 \% \\
\text { HRmax. } \\
\text { IT (MVIT): } 30 \text { s of "all out" } \\
\text { exercise followed by } 4 \text { min } \\
\text { of low intensity recovery. }\end{array}$ & $\begin{array}{l}\text { MICT: } 3 \times \\
\text { IT (AIT): } 3 \times \\
\text { IT (MVIT): } \\
3 \times\end{array}$ & $\begin{array}{l}\text { MICT: } 48 \mathrm{~min} \\
\text { IT (AIT): } \\
40 \mathrm{~min} \\
\text { IT (MVIT): } \\
24.5-40 \mathrm{~min}\end{array}$ & BIA \\
\hline$[64]$ & $\begin{array}{l}\text { Healthy, } \\
\text { recreation- } \\
\text { ally active } \\
\text { young adults } \\
\text { (mean age: } \\
23 \text { years) }\end{array}$ & 6 & $\begin{array}{l}\text { MICT: } 10 \\
\text { IT: } 10\end{array}$ & $\begin{array}{l}\text { MICT: running, } 65 \% \text { of } \\
\text { VO } \mathrm{V}_{2} \mathrm{max} \\
\text { IT: } 4-6 \text { bouts of } 30 \mathrm{~s} \\
\text { maximal running efforts } \\
\text { with } 4 \text { min of recovery } \\
\text { (active recovery } \\
\text { encouraged) }\end{array}$ & $\begin{array}{l}\text { MICT: } 3 \times \\
\text { IT: } 3 \times\end{array}$ & $\begin{array}{l}\text { MICT: } \\
\text { 30-60 min } \\
\text { IT: } 18-27 \mathrm{~min}\end{array}$ & ADP \\
\hline [65] & $\begin{array}{l}\text { Overweight, } \\
\text { untrained } \\
\text { men (aged } \\
28-46 \text { years) }\end{array}$ & 10 & $\begin{array}{l}\text { MICT: } 7 \\
\text { IT: } 7\end{array}$ & $\begin{array}{l}\text { MICT: } 50 \% \text { of } \mathrm{VO}_{2} \max \\
\text { IT: } 25 \text { sets of } 80 \mathrm{~s} \text { at } 35 \% \\
\mathrm{VO}_{2} \text { max followed by } 40 \mathrm{~s} \\
\text { at } 80 \% \mathrm{VO}_{2} \text { max. }\end{array}$ & $\begin{array}{l}\text { MICT: } 3 \times \\
\text { IT: } 3 \times\end{array}$ & $\begin{array}{l}\text { MICT: } 50 \mathrm{~min} \\
\text { IT: } 50 \mathrm{~min}\end{array}$ & Skinfolds \\
\hline [66] & $\begin{array}{c}\text { Adults with } \\
\text { type } 2 \\
\text { diabetes } \\
\text { (mean age: } \\
59 \text { years) }\end{array}$ & 52 & $\begin{array}{l}\text { MICT: } 24 \\
\text { IT: } 19 \\
\text { CONT: } 24\end{array}$ & $\begin{array}{c}\text { MICT: cycling } 40-60 \% \text { of } \\
\text { HRR } \\
\text { IT: cycling, } 2 \text { min at } \\
\text { 70-80\% of HRR with } 1 \\
\text { min at } 40-60 \% \text { of HRR. } 1 \\
\text { min at } 90 \% \text { of HRR with } 1 \\
\text { min resting at } 40-60 \% \text { of } \\
\text { HRR. }\end{array}$ & $\begin{array}{l}\text { MICT: } 3 \times \\
\text { IT: } 3 \times\end{array}$ & $\begin{array}{c}\text { MICT: } 45 \pm \\
7.1 \mathrm{~min} \\
\text { IT: } \\
33.1 \pm 6.4 \mathrm{~min}\end{array}$ & DXA \\
\hline [67] & $\begin{array}{l}\text { Postmenopausal } \\
\text { women with } \\
\text { type } 2 \\
\text { diabetes } \\
\text { (mean age: } \\
69 \text { years) }\end{array}$ & I & $\begin{array}{l}\text { MICT: } 8 \\
\text { IT: } 8\end{array}$ & $\begin{array}{c}\text { MICT: } 55-60 \% \text { of } \\
\text { individual HR reserve } \\
\text { IT: } 60 \text { cycle (maximum) of } \\
8 \mathrm{~s} \text { at } 77-85 \% \text { HRmax with } \\
\text { active recovery of } 20-30 \\
\text { rpm for } 12 \mathrm{~s} .\end{array}$ & $\begin{array}{l}\text { MICT: } 2 \times \\
\text { IT: } 2 \times\end{array}$ & $\begin{array}{l}\text { MICT: } 40 \mathrm{~min} \\
\text { IT: } 25 \mathrm{~min}\end{array}$ & DXA \\
\hline [68] & $\begin{array}{l}\text { Children } \\
\text { (aged 7-9 } \\
\text { years) }\end{array}$ & 12 & $\begin{array}{l}\text { MICT: } 56 \\
\text { IT: } 38\end{array}$ & $\begin{array}{c}\text { MICT: } 20 \text { min of } \\
\text { moderate-intensity } \\
\text { aerobic exercises and } \\
\text { games followed by } 20 \mathrm{~min} \\
\text { of sport. } \\
\text { IT: } 20 \text { min of } 10-20 \mathrm{~s} \text { of } \\
\text { high-intensity intermittent } \\
\text { exercises followed by } 20 \\
\text { min of sports activities. }\end{array}$ & $\begin{array}{l}\text { MICT: } 2 \times \\
\text { IT: } 2 \times\end{array}$ & $\begin{array}{l}\text { MICT: } 40 \mathrm{~min} \\
\text { IT: } 40 \mathrm{~min}\end{array}$ & BIA \\
\hline
\end{tabular}


Table 1. Cont

\begin{tabular}{|c|c|c|c|c|c|c|c|}
\hline Study & $\begin{array}{c}\text { Sample } \\
\text { Population } \\
\text { (age) }\end{array}$ & $\begin{array}{l}\text { Duration } \\
\text { (weeks) }\end{array}$ & Group (n) & Modality/Intensity & $\begin{array}{l}\text { Frequency } \\
\text { (weeks) }\end{array}$ & $\begin{array}{l}\text { Time per } \\
\text { Session }\end{array}$ & $\begin{array}{c}\text { Body } \\
\text { Composition } \\
\text { Method }\end{array}$ \\
\hline [69] & $\begin{array}{l}\text { Sedentary } \\
\text { Obese Adults } \\
\text { (aged } 34 \\
\text { years) }\end{array}$ & 12 & $\begin{array}{l}\text { MICT: } 14 \\
\text { IT: } 16 \\
\frac{1}{2} \text { IT: } 16\end{array}$ & $\begin{array}{l}\text { MICT: } 70 \% \text { of peak HR } \\
\text { IT: } 8 \text { s of maximal } \\
\text { intensity sprint intervals } \\
\text { on a bike at } 85-90 \% \text { of } \\
\text { peak HR, with } 12 \text { s rest } \\
\text { intervals pedaling as slow } \\
\text { as possible. Sequence } \\
\text { continued until the } 250 \\
\text { kcal target was met. } \\
\frac{1}{2} \text { IT: Same as IT but with a } \\
125 \text { kcal target. }\end{array}$ & $\begin{array}{c}\text { MICT: } 3 \times \\
\text { IT: } 3 \times \\
\frac{1}{2} \text { IT: } 3 \times\end{array}$ & $\begin{array}{l}\text { MICT: } 32 \\
\text { min(avg.) } \\
\text { IT: } 20 \\
\text { min(avg.) } \\
\frac{1}{2} \text { IT: } 10 \text { min } \\
\text { (avg.) }\end{array}$ & DXA \\
\hline$[70]$ & $\begin{array}{l}\text { Sedentary } \\
\text { adult men } \\
\text { (aged } 29 \\
\text { years) }\end{array}$ & 8 & $\begin{array}{l}\text { MICT: } 12 \\
\text { IT: } 12\end{array}$ & $\begin{array}{l}\text { MICT: } 60-65 \% \mathrm{VO}_{2} \max \\
\text { IT: } 3,3 \text { min intervals of } \\
\text { high intensity cycling at } \\
80-85 \% \mathrm{VO}_{2} \text { max with } 2 \\
\text { active rest intervals. }\end{array}$ & $\begin{array}{l}\text { MICT: } 3 \times \\
\text { IT: } 3 \times\end{array}$ & $\begin{array}{l}\text { MICT: } 45 \text { min } \\
\text { IT: } 18 \text { min } \\
\text { total } \\
\text { including } \\
5 \text { min of } \\
\text { combined } \\
\text { warm-up and } \\
\text { cool down. }\end{array}$ & DXA \\
\hline [71] & $\begin{array}{l}\text { Adult men } \\
\text { with } \\
\text { metabolic } \\
\text { syndrome } \\
\text { (mean age: } \\
48 \text { years) }\end{array}$ & 8 & $\begin{array}{l}\text { MICT: } 13 \\
\text { IT: } 13\end{array}$ & $\begin{array}{l}\text { MICT: cycling at } 60-65 \% \\
\text { of } \mathrm{VO}_{2} \text { peak } \\
\text { IT: } 3 \text { sets of } 3 \text { min cycling } \\
\text { at } 80-85 \% \mathrm{VO}_{2} \text { peak with a } \\
2 \text { min active rest between } \\
\text { sets at } 50 \% \mathrm{VO}_{2} \text { peak }\end{array}$ & $\begin{array}{l}\text { MICT: } 3 \times \\
\text { IT: } 3 \times\end{array}$ & $\begin{array}{l}\text { MICT: } 45 \mathrm{~min} \\
\text { IT: } 18 \mathrm{~min}\end{array}$ & DXA \\
\hline [72] & $\begin{array}{l}\text { Sedentary } \\
\text { pre- } \\
\text { menopausal } \\
\text { women } \\
\text { (mean age: } \\
45 \text { years) }\end{array}$ & 15 & $\begin{array}{l}\text { MICT: } 21 \\
\text { IT: } 21 \\
\text { CON: } 20\end{array}$ & $\begin{array}{l}\text { MICT: Moderate intensity } \\
\text { swimming at } \sim 70 \% \\
\text { HRmax. } \\
\text { IT: } 6-10 \times 30 \mathrm{~s} \text { all-out } \\
\text { swimming with } 2 \text { min } \\
\text { recovery in between each } \\
\text { bout at. } \sim 90 \% \text { HRmax }\end{array}$ & $\begin{array}{l}\text { MICT: } 3 \times \\
\text { IT: } 3 \times\end{array}$ & $\begin{array}{l}\text { MICT: } 1 \mathrm{~h} \\
\text { IT: } 15-25 \mathrm{~min} \\
\text { total. }\end{array}$ & DXA \\
\hline [73] & $\begin{array}{l}\text { Overweight } \\
\text { adults (mean } \\
\text { age: } 40 \text { years) }\end{array}$ & 12 & $\begin{array}{l}\text { MICT: } 8 \\
\text { IT: } 8 \\
\text { CON: } 7\end{array}$ & $\begin{array}{l}\text { MICT: Biking at } 10 \% \text { lower } \\
\text { than anaerobic threshold. } \\
\text { IT: Biking at } 20 \% \text { above } \\
\text { anaerobic threshold with } \\
\text { an exercise:pause ratio of } \\
\text { 2:1. }\end{array}$ & $\begin{array}{l}\text { MICT: } 3 \times \\
\text { IT: } 3 \times\end{array}$ & $\begin{array}{l}\text { Both groups } \\
\text { completed } \\
20 \text { min in the } \\
\text { first week, } \\
\text { with } \\
\text { increments of } \\
10 \text { min per } \\
\text { week until a } \\
\text { total of } \\
60 \text { min per } \\
\text { session was } \\
\text { reached in } \\
\text { the fourth } \\
\text { week. }\end{array}$ & BIA \\
\hline
\end{tabular}


Table 1. Cont.

\begin{tabular}{|c|c|c|c|c|c|c|c|}
\hline Study & $\begin{array}{c}\text { Sample } \\
\text { Population } \\
\text { (age) }\end{array}$ & $\begin{array}{l}\text { Duration } \\
\text { (weeks) }\end{array}$ & Group (n) & Modality/Intensity & $\begin{array}{l}\text { Frequency } \\
\text { (weeks) }\end{array}$ & $\begin{array}{l}\text { Time per } \\
\text { Session }\end{array}$ & $\begin{array}{c}\text { Body } \\
\text { Composition } \\
\text { Method }\end{array}$ \\
\hline [74] & $\begin{array}{c}\text { Obese } \\
\text { adolescents } \\
\text { (mean age: } \\
15 \text { years) }\end{array}$ & 12 & $\begin{array}{l}\text { MICT: } 13 \\
\text { IT: } 16\end{array}$ & $\begin{array}{l}\text { MICT: Boxing and Nordic } \\
\text { walking at } 60-75 \% \text { of } \\
\text { maximal HR. } \\
\text { IT: } 4 \text { to } 6 \text { intervals of } 2 \\
\text { min- } 2 \text { min } 30 \mathrm{~s} \text { in } \\
\text { duration at } 90-95 \% \text { of } \\
\text { HRmax interspersed by } 1 \\
\text { min } 30 \text { s intervals at } 55 \% \\
\text { of HRmax }\end{array}$ & $\begin{array}{l}\text { MICT: } 3 \times \\
\text { IT: } 3 \times\end{array}$ & $\begin{array}{l}\text { MICT: } 40 \text { to } \\
60 \text { min. } \\
\text { IT: } 24 \text { to } \\
32 \text { mins }\end{array}$ & DXA \\
\hline [75] & $\begin{array}{c}\text { Obese } \\
\text { adolescents } \\
\text { (mean age: } \\
14 \text { years) }\end{array}$ & 4 & $\begin{array}{l}\text { MICT: } 8 \\
\text { IT: } 10\end{array}$ & $\begin{array}{l}\text { MICT: } 65 \% \text { HRmax } \\
\text { IT: } 1 \text { min vigorous } \\
\text { treadmill exercise at } 80 \% \\
\text { to } 90 \% \text { HRmax } \\
\text { interspersed with } 2 \text { min } \\
\text { recovery intervals at } 60 \% \\
\text { HRmax }\end{array}$ & $\begin{array}{l}\text { MICT: } 3 \times \\
\text { IT: } 3 \times\end{array}$ & MICT: $50 \mathrm{~min}$ & BIA \\
\hline [76] & $\begin{array}{l}\text { Recreationally } \\
\text { active men } \\
\text { (mean age: } \\
21.7 \text { years) }\end{array}$ & 7 & $\begin{array}{l}\text { MICT: } 7 \\
\text { IT: } 8\end{array}$ & $\begin{array}{l}\text { MICT: Cycling at } 60 \% \text { of } \\
\mathrm{VO}_{2} \text { max. } \\
\text { IT: } 4-6 \text { Wingate sprints } \\
\text { (resistance }=7.5 \% \text { of } \\
\text { subject } \mathrm{BW} \text { ) with } 4.5 \mathrm{~min} \\
\text { recovery }\end{array}$ & $\begin{array}{l}\text { MICT: } 3 \times \\
\text { IT: } 3 \times\end{array}$ & $\begin{array}{l}\text { MICT: } \\
\text { 30-50 min } \\
\text { IT: } 30 \mathrm{~min}\end{array}$ & Skinfolds \\
\hline [77] & $\begin{array}{c}\text { Untrained } \\
\text { men (mean } \\
\text { age: } 33 \text { years) }\end{array}$ & 12 & $\begin{array}{c}\text { MICT: } 9 \\
\text { IT; } 8 \\
\text { CON:11 }\end{array}$ & $\begin{array}{l}\text { MICT: } 80 \% \text { HRmax } \\
\text { IT: Five intervals of } 2 \text { min } \\
\text { of near-maximal running } \\
\text { (HR above } 95 \% \text { of their } \\
\text { HRmax at the end of the } 2 \\
\text { min period interspersed } \\
\text { by } 1 \text { min rest. }\end{array}$ & $\begin{array}{l}\text { MICT: } 3 \times \\
\text { IT: } 2 \times \\
\text { (attempted } 3 \\
\text { but accom- } \\
\text { plished } 2 \text { on } \\
\text { average due } \\
\text { to injuries or } \\
\text { other } \\
\text { reasons) }\end{array}$ & $\begin{array}{l}\text { MICT: } 1 \mathrm{~h} \\
\text { IT: } 20 \mathrm{~min}\end{array}$ & DXA \\
\hline [78] & $\begin{array}{c}\text { Sedentary } \\
\text { obese males } \\
\text { (mean age: } \\
48.4 \text { years) }\end{array}$ & 12 & $\begin{array}{l}\text { MICT: } 13 \\
\text { IT: } 20\end{array}$ & $\begin{array}{l}\text { MICT: Cycling at } 60-65 \% \\
\text { VO }_{2} \text { max } \\
\text { IT: } 3 \text { sets of } 180 \text { s cycling at } \\
80-85 \% \mathrm{VO}_{2} \max \text { with } 120 \\
\text { s recovery period at } 50 \% \\
\mathrm{VO}_{2} \max \end{array}$ & $\begin{array}{l}\text { MICT: } 3 \times \\
\text { IT: } 3 \times\end{array}$ & $\begin{array}{l}\text { MICT: } 40 \mathrm{~min} \\
\text { IT } 13 \mathrm{~min}\end{array}$ & DXA \\
\hline [79] & $\begin{array}{l}\text { Untrained } \\
\text { women } \\
\text { (mean age: } \\
28.4 \text { years) }\end{array}$ & 6 & $\begin{array}{l}\text { MICT: } 12 \\
\text { IT: } 11\end{array}$ & $\begin{array}{l}\text { MICT: Cycling at } 70 \% \\
\text { HRmax } \\
\text { IT: Cycling } 15 \text { sets } 60 \mathrm{~s} \text { at } \\
\text { 90\% HRmax with } 30 \mathrm{~s} \\
\text { recovery period at } 60 \% \\
\text { HRmax }\end{array}$ & $\begin{array}{l}\text { MICT: } 3 \times \\
\text { IT: } 3 \times\end{array}$ & $\begin{array}{l}\text { MICT: } 29 \min \\
\text { IT: } 22 \mathrm{~min}\end{array}$ & Skinfolds \\
\hline [80] & $\begin{array}{l}\text { Untrained } \\
\text { obese women } \\
\text { (mean age: } \\
46 \text { years) }\end{array}$ & 12 & $\begin{array}{l}\text { MICT: } 12 \\
\text { IT: } 18\end{array}$ & $\begin{array}{l}\text { MICT: Deep water } \\
\text { running at } 65-85 \% \text { HRR } \\
\text { IT: Deep water running } \\
\text { 8-15, } 15 \text { s sprints with } 30 \mathrm{~s} \\
\text { recovery interspersed } \\
\text { with } 5-14 \text { min intervals at } \\
70-75 \% \text { HRmax }\end{array}$ & $\begin{array}{l}\text { MICT: } 3 \times \\
\text { IT: } 3 \times\end{array}$ & $\begin{array}{l}\text { MICT: } 47 \mathrm{~min} \\
\text { IT: } 47 \mathrm{~min} \\
\text { (including } \\
\text { recovery } \\
\text { periods) }\end{array}$ & Skinfolds \\
\hline
\end{tabular}


Table 1. Cont.

\begin{tabular}{|c|c|c|c|c|c|c|c|}
\hline Study & $\begin{array}{c}\text { Sample } \\
\text { Population } \\
\text { (age) }\end{array}$ & $\begin{array}{c}\text { Duration } \\
\text { (weeks) }\end{array}$ & Group (n) & Modality/Intensity & $\begin{array}{l}\text { Frequency } \\
\text { (weeks) }\end{array}$ & $\begin{array}{l}\text { Time per } \\
\text { Session }\end{array}$ & $\begin{array}{c}\text { Body } \\
\text { Composition } \\
\text { Method }\end{array}$ \\
\hline [81] & $\begin{array}{c}\text { Healthy } \\
\text { physically } \\
\text { inactive } \\
\text { adults (mean } \\
\text { age: } 32 \text { years) }\end{array}$ & 12 & $\begin{array}{c}\text { MICT: } 9 \\
\text { IT: } 11\end{array}$ & $\begin{array}{c}\text { MICT: Treadmill, } 60-80 \% \\
\text { HRR } \\
\text { IT: Treadmill, } 4 \text { sets } 240 \mathrm{~s} \\
\text { at } 85-95 \% \text { peak HRR with } \\
240 \text { s recovery period at } \\
65 \% \text { peak HRR }\end{array}$ & $\begin{array}{l}\text { MICT: } 3 \times \\
\text { IT: } 3 \times\end{array}$ & $\begin{array}{c}\text { MICT: } \\
\text { 20-65 min } \\
\text { (including } \\
\text { warm up and } \\
\text { cool down) } \\
\text { IT: } 35 \text { to } \\
55 \text { min } \\
\text { (including } \\
\text { warm up and } \\
\text { cool down) }\end{array}$ & BIA \\
\hline
\end{tabular}

\begin{tabular}{|c|c|c|c|c|c|c|c|}
\hline [82] & $\begin{array}{l}\text { Adults with } \\
\text { metabolic } \\
\text { syndrome } \\
\text { (mean age: } 57 \\
\text { years) }\end{array}$ & 16 & $\begin{array}{l}\text { MICT: } 21 \\
\text { IT (a): } 22 \\
\text { IT (b): } 23\end{array}$ & $\begin{array}{l}\text { MICT: Cycling, } 60-70 \% \text { of } \\
\text { peak heart rate } \\
\text { IT (a) Cycling, } 4 \mathrm{HIIT} \\
\text { group-4 } 240 \mathrm{~s} \text { sets at } \\
85-95 \% \text { peak heart rate } \\
\text { with } 180 \mathrm{~s} \text { recovery period } \\
\text { at } 50-70 \% \text { peak heart rate } \\
\text { IT (b) Cycling, } 1 \mathrm{HIIT} \\
\text { group- } 1 \text { set } 240 \mathrm{~s} \text { at } \\
85-95 \% \text { peak heart rate } \\
\text { with } 180 \text { s cool down at } \\
60-70 \% \text { peak heart rate }\end{array}$ & $\begin{array}{l}\text { MICT: } 5 \times \\
\text { IT (a): } 3 \times \\
\text { IT (b): } 3 \times\end{array}$ & $\begin{array}{l}\text { MICT: } 30 \text { min } \\
\text { IT (a): } 4 \text { HIIT, } \\
38 \text { min } \\
\text { (including } \\
\text { warm up and } \\
\text { cool down). } \\
\text { IT (b): } 1 \text { HIIT, } \\
17 \text { min } \\
\text { (including } \\
\text { warm up and } \\
\text { cool down) }\end{array}$ & DXA \\
\hline [83] & $\begin{array}{c}\text { Sedentary } \\
\text { adults (mean } \\
\text { age: } 31 \text { years) }\end{array}$ & 8 & $\begin{array}{l}\text { MICT: } 7 \\
\text { IT (a): } 9 \\
\text { IT (b): } 11\end{array}$ & $\begin{array}{c}\text { MICT: Cycling, } 65-75 \% \\
\text { HRmax } \\
\text { IT (a): } 2 \times 4 \text { HIIT, cycling } \\
2 \text { sets } 240 \text { s at } 85-95 \% \\
\text { HRMax with } 120 \text { s active } \\
\text { rest } \\
\text { IT (b): } 5 \times 1 \text { HIIT, cycling } \\
5 \text { sets } 60 \text { s at } 85-95 \% \\
\text { HRMax with } 60 \text { s active } \\
\text { rest }\end{array}$ & $\begin{array}{l}\text { MICT: } 2 \times \\
\text { IT (a): } 2 \times \\
\text { IT (b): } 2 \times\end{array}$ & $\begin{array}{l}\text { MICT: } 38 \text { min } \\
\text { (including } \\
\text { warm up and } \\
\text { cool down) } \\
\text { IT (a): } 15 \text { min } \\
\text { (including } \\
\text { warm up and } \\
\text { cool down) } \\
\text { IT (b): } 14 \text { min } \\
\text { (Including } \\
\text { warm up and } \\
\text { cool down) }\end{array}$ & BIA \\
\hline [84] & $\begin{array}{c}\text { Sedentary } \\
\text { males (age } \\
\text { not reported) }\end{array}$ & 4 & $\begin{array}{l}\text { MICT:12 } \\
\text { IT: } 12\end{array}$ & $\begin{array}{l}\text { MICT: Cycling, } 45 \% \\
\mathrm{VO}_{2} \max \\
\text { IT: Cycling, } 10 \text { sets, } 60 \mathrm{~s} \text { at } \\
85 \% \mathrm{VO}_{2} \text { max with } 30 \mathrm{~s} \\
\text { rest period between sets }\end{array}$ & $\begin{array}{c}\text { MICT:3× } \\
\text { IT: } 3 \times\end{array}$ & $\begin{array}{l}\text { MICT: } 22 \text { min } \\
\text { IT: } 15 \text { min } \\
\text { (including } \\
\text { rest periods) }\end{array}$ & BIA \\
\hline [85] & $\begin{array}{l}\text { Obese adults } \\
\text { (mean age: } \\
46 \text { years) }\end{array}$ & 12 & $\begin{array}{l}\text { MICT: } 13 \\
\text { IT:14 }\end{array}$ & $\begin{array}{l}\text { MICT: Treadmill, } 60-70 \% \\
\text { HRMax } \\
\text { IT: Treadmill, } 4 \text { sets } 240 \mathrm{~s} \\
\text { at } 85-95 \% \text { HRMax with } \\
180 \mathrm{~s} \text { rest periods at } \\
50-60 \% \text { HRMax }\end{array}$ & $\begin{array}{l}\text { MICT:3× } \\
\text { IT: } 3 \times\end{array}$ & $\begin{array}{l}\text { MICT: } 47 \mathrm{~min} \\
\text { IT: } 42 \mathrm{~min} \\
\text { (including } \\
\text { warm up and } \\
\text { cool down) }\end{array}$ & DXA \\
\hline [86] & $\begin{array}{c}\text { Sedentary } \\
\text { males (mean } \\
\text { age: } 2 \text { years) }\end{array}$ & 6 & $\begin{array}{c}\text { MICT: } 8 \\
\text { IT: } 8\end{array}$ & $\begin{array}{l}\text { MICT: Cycling, 65\% } \\
\text { VO }_{2} \text { Peak } \\
\text { IT: Cycling, four to six, } \\
30 \mathrm{~s} \text { 'all out' sprints } \\
\text { (Wingate test) with } 270 \mathrm{~s} \\
\text { rest between each test }\end{array}$ & $\begin{array}{c}\text { MICT:5× } \\
\text { IT: } 3 \times\end{array}$ & $\begin{array}{l}\text { MICT: } 40 \text { to } \\
\quad 60 \mathrm{~min} \\
\text { IT: } 20-30 \mathrm{~min} \\
\text { (including } \\
\text { rest periods) }\end{array}$ & DXA \\
\hline
\end{tabular}


Table 1. Cont

\begin{tabular}{|c|c|c|c|c|c|c|c|}
\hline Study & $\begin{array}{c}\text { Sample } \\
\text { Population } \\
\text { (age) }\end{array}$ & $\begin{array}{c}\text { Duration } \\
\text { (weeks) }\end{array}$ & Group (n) & Modality/Intensity & $\begin{array}{l}\text { Frequency } \\
\text { (weeks) }\end{array}$ & $\begin{array}{l}\text { Time per } \\
\text { Session }\end{array}$ & $\begin{array}{c}\text { Body } \\
\text { Composition } \\
\text { Method }\end{array}$ \\
\hline [87] & $\begin{array}{l}\text { Overweight } \\
\text { adults (mean } \\
\text { age: } 42 \text { years) }\end{array}$ & 10 & $\begin{array}{l}\text { MICT: } 44 \\
\text { IT: } 46\end{array}$ & $\begin{array}{l}\text { MICT: Cycling, } 70 \% \\
\text { MHR } \\
\text { IT: Cycling, }>90 \% \text { MHR, } \\
\text { repeated sprints of } 15-60 \mathrm{~s}, \\
\text { interspersed with periods } \\
\text { of recovery cycling }\end{array}$ & $\begin{array}{l}\text { MICT: } 5 \times \\
\text { IT: } 3 \times\end{array}$ & $\begin{array}{c}\text { MICT: } \\
\text { 30-45 min } \\
\text { IT: } 18-25 \mathrm{~min}\end{array}$ & BIA \\
\hline [88] & $\begin{array}{l}\text { Trained } \\
\text { young adults } \\
\text { (mean age: } \\
19 \text { years) }\end{array}$ & 8 & $\begin{array}{c}\text { MICT: } 7 \\
\text { IT: } 7\end{array}$ & $\begin{array}{l}\text { MICT: Rowing, Blood } \\
\text { Lactate Concentrations of } \\
\quad 2-3 \text { mmol/L } \\
\text { IT: Rowing, eight, } 2.5 \text { min } \\
\text { intervals at } 90 \% \text { of mean } 4 \\
\text { min maximal power } \\
\text { output achieved during } \\
\text { the incremental exercise } \\
\text { test. Recovery duration } \\
\text { was until HR returned } \\
70 \% \text { MHR, at } 40 \% \text { of mean } \\
\text { maximal power output }\end{array}$ & $\begin{array}{l}\text { MICT: } 2 \times \\
\text { IT: } 2 \times\end{array}$ & $\begin{array}{c}\text { MICT: } \\
\text { 35/40 min } \\
\text { IT: } 27-55 \text { min } \\
\text { (including } \\
\text { recovery) }\end{array}$ & DXA \\
\hline [89] & $\begin{array}{l}\text { Overweight } \\
\text { Young } \\
\text { Adults (mean } \\
\text { age: } 20 \text { years) }\end{array}$ & 12 & $\begin{array}{l}\text { MICT: } 16 \\
\text { IT: } 17 \\
\text { CON: } 19\end{array}$ & $\begin{array}{l}\text { MICT: Walking/Jogging, } \\
\mathrm{HR} \text { associated with } 50 \% \text { of } \\
\mathrm{VO}_{2} \mathrm{max} \\
\text { IT: Running, five, } 3 \mathrm{~min} \\
\text { intervals at the } \mathrm{HR} \\
\text { associated with } 85 \% \\
\mathrm{VO}_{2} \text { max with } 3 \text { min active } \\
\text { rest at } \mathrm{HR} \text { associated } 50 \% \\
\mathrm{VO}_{2} \text { max between each } \\
\text { interval }\end{array}$ & $\begin{array}{l}\text { MICT: } 5 \times \\
\text { IT: } 5 \times\end{array}$ & $\begin{array}{l}\text { MICT: } 55 \mathrm{~min} \\
\text { IT: } 42 \mathrm{~min} \\
\text { (Including } \\
\text { warm up and } \\
\text { cool down) }\end{array}$ & DXA \\
\hline [90] & $\begin{array}{l}\text { Overweight } \\
\text { Males (mean } \\
\text { age: } 31 \text { yrs) }\end{array}$ & 12 & $\begin{array}{l}\text { MICT: } 10 \\
\text { IT: } 10 \\
\text { CON: } 10\end{array}$ & $\begin{array}{l}\text { MICT: Cycling, } ~ 60 \% \\
\mathrm{VO}_{2} \text { peak } \\
\text { IT: Cycling, } 15 \mathrm{~s} \text { at a } \\
\text { power output equivalent } \\
\text { to } \sim 170 \% \mathrm{VO}_{2} \text { peak with } \\
\text { an active recovery period } \\
\text { of } 60 \mathrm{~s} \text { at a power output } \\
\text { equivalent to } \sim 32 \% \\
\mathrm{VO}_{2} \text { peak } \\
\text { Relative total work was } \\
\text { matched between both } \\
\text { groups }\end{array}$ & $\begin{array}{l}\text { MICT: } 3 \times \\
\text { IT: } 3 \times\end{array}$ & $\begin{array}{c}\text { MICT: } \\
\text { 30-45 min } \\
\text { IT: } 30-45 \mathrm{~min}\end{array}$ & DXA \\
\hline [91] & $\begin{array}{l}\text { Obese } \\
\text { Children } \\
\text { (mean age: } \\
15 \text { years) }\end{array}$ & 6 & $\begin{array}{l}\text { MICT: } 13 \\
\text { IT: } 14\end{array}$ & $\begin{array}{l}\text { MICT: Cycling, } 65-70 \% \\
\text { APMHR } \\
\text { IT: Cycling, ten, } 2 \text { min } \\
\text { bouts at } 90-95 \% \text { APMHR, } \\
\text { with } 1 \text { min of active } \\
\text { recovery at } 55 \% \text { APMHR } \\
\text { between each bout }\end{array}$ & $\begin{array}{l}\text { MICT: } 3 \times \\
\text { IT: } 3 \times\end{array}$ & $\begin{array}{l}\text { MICT: } 40 \mathrm{~min} \\
\text { IT: } 40 \text { min } \\
\text { (Including } \\
\text { warmup and } \\
\text { cool down) }\end{array}$ & ADP \\
\hline
\end{tabular}


Table 1. Cont.

\begin{tabular}{|c|c|c|c|c|c|c|c|}
\hline Study & $\begin{array}{c}\text { Sample } \\
\text { Population } \\
\text { (age) }\end{array}$ & $\begin{array}{l}\text { Duration } \\
\text { (weeks) }\end{array}$ & Group (n) & Modality/Intensity & $\begin{array}{l}\text { Frequency } \\
\text { (weeks) }\end{array}$ & $\begin{array}{l}\text { Time per } \\
\text { Session }\end{array}$ & $\begin{array}{c}\text { Body } \\
\text { Composition } \\
\text { Method }\end{array}$ \\
\hline [92] & $\begin{array}{c}\text { Healthy } \\
\text { Untrained } \\
\text { Adults (aged } \\
\text { 18-32 years) }\end{array}$ & 12 & $\begin{array}{l}\text { MICT A: } 14 \\
\text { MICT B: } 18 \\
\text { IT: } 15\end{array}$ & $\begin{array}{c}\text { MICT A: Running, } 4 \text { m, } \\
75 \% \text { MHR } \\
\text { MICT B: Running, } 2 \text { m, } \\
75 \% \text { MHR } \\
\text { IT: Running, } 8 \text { bouts of } \\
60 \text { s intervals at } 90 \% \text { MHR } \\
\text { followed by } 180 \text { s rest } \\
\text { between each bout }\end{array}$ & $\begin{array}{l}\text { MICT: } 3 \times \\
\text { IT: } 3 \times\end{array}$ & $\begin{array}{l}\text { MICT A: } \\
\sim 32 \mathrm{~min} / \sim 500 \\
\mathrm{cal} / \mathrm{session} \\
\mathrm{MICT} \mathrm{B}: \sim 16 \\
\mathrm{~min} / \sim 250 \\
\mathrm{cal} / \mathrm{session} \\
\text { IT: } 29 \mathrm{~min}\end{array}$ & $\begin{array}{l}\text { Hydrostatic } \\
\text { densitometry }\end{array}$ \\
\hline [93] & $\begin{array}{l}\text { Healthy } \\
\text { Inactive } \\
\text { Young } \\
\text { Females } \\
\text { (mean age: } 21 \\
\text { years) }\end{array}$ & 15 & $\begin{array}{l}\text { MICT: } 15 \\
\text { IT: } 15\end{array}$ & $\begin{array}{l}\text { MICT: Cycling, } 60 \% \\
\mathrm{VO}_{2} \text { peak } \\
\text { IT: Cycling, maximum of } \\
60 \text { bouts of } 8 \mathrm{~s}: 12 \mathrm{~s} \text { ratio of } \\
\text { sprinting and slow } \\
\text { pedaling }\end{array}$ & $\begin{array}{l}\text { MICT: } 3 \times \\
\text { IT: } 3 \times\end{array}$ & $\begin{array}{l}\text { MICT: } \\
\text { 20-50 min } \\
\text { IT: } 15-30 \mathrm{~min} \\
\text { (Including } \\
\text { warmup and } \\
\text { cool down }\end{array}$ & DXA \\
\hline [94] & $\begin{array}{l}\text { Obese Adults } \\
\text { (mean age: } \\
43 \text { years) }\end{array}$ & 8 & $\begin{array}{l}\text { MICT: } 6 \\
\text { IT: } 7 \\
\text { CON: } 8\end{array}$ & $\begin{array}{c}\text { MICT: Cycling, } 50-65 \% \\
\mathrm{VO}_{2} \text { peak } \\
\text { IT: } 2 \text { min ratio of high to } \\
\text { low intensity of } 90-105 \% \\
\mathrm{VO}_{2} \text { peak and } 30-45 \% \\
\mathrm{VO}_{2} \text { peak }\end{array}$ & $\begin{array}{l}\text { MICT: } 4 \times \\
\text { IT: } 4 \times\end{array}$ & $\begin{array}{l}\text { MICT: } 30 \mathrm{~min} \\
\text { IT:30 min } \\
\text { (Including } \\
\text { recovery) }\end{array}$ & DXA \\
\hline [95] & $\begin{array}{l}\text { Overweight } \\
\text { Adults (mean } \\
\text { age: } 56 \text { years) }\end{array}$ & 11 & $\begin{array}{l}\text { MICT: } 12 \\
\text { IT: } 13 \\
\text { CON: } 7\end{array}$ & $\begin{array}{c}\text { MICT: Cycling, 50\% } \\
\text { Wpeak } \\
\text { IT: Cycling, } 1 \mathrm{~min} \text { at } 95 \% \\
\text { Wpeak, with } 1 \mathrm{~min} \text { active } \\
\text { recovery at } 20 \% \text { Wpeak } \\
\text { between each bout. }\end{array}$ & $\begin{array}{l}\text { MICT: } 3 \times \\
\text { IT: } 3 \times\end{array}$ & $\begin{array}{c}\text { MICT: } \\
135 \mathrm{~min} \\
\text { IT: } 75 \mathrm{~min} \\
\text { (Including } \\
\text { the warmup) }\end{array}$ & DXA \\
\hline [96] & $\begin{array}{l}\text { Overweight } \\
\text { Young } \\
\text { Females } \\
\text { (mean age: } \\
21 \text { years) }\end{array}$ & 12 & $\begin{array}{l}\text { MICT: } 15 \\
\text { IT: } 15 \\
\text { CON: } 13\end{array}$ & $\begin{array}{c}\text { MICT: Cycling, } 60 \% \\
\mathrm{VO}_{2} \text { max until } 300 \mathrm{~kJ} \text { of } \\
\text { work is reached } \\
\text { IT: Cycling, repeated } \\
4 \text { min bouts at } 90 \% \\
\mathrm{VO}_{2} \text { max with } 3 \mathrm{~min} \\
\text { passive recovery between } \\
\text { bouts until } 300 \mathrm{~kJ} \text { of work } \\
\text { is reached }\end{array}$ & $\begin{array}{c}\text { MICT: } 3-4 \times \\
\text { IT: } 3-4 \times\end{array}$ & $\begin{array}{l}\text { MICT: Until } \\
300 \mathrm{~kJ} \text { of } \\
\text { work was } \\
\text { reached } \\
\text { IT: Until m of } \\
\text { work was } \\
\text { reached }\end{array}$ & DXA \\
\hline
\end{tabular}

Abbreviations: $\mathrm{MICT}=$ moderate intensity continuous training; $\mathrm{IT}=$ interval training; $\mathrm{CON}=$ control; $\mathrm{BIA}=$ bioelectrical impedance analysis; DXA = dual energy $\mathrm{x}$-ray absorptiometry; $\mathrm{ADP}$ = air displacement plethysmography.

\subsection{Methodological Quality}

Study quality, as assessed by the PEDro scale, had a mean rating of 5.6, indicating that the overall pool of studies are of good quality. A total of 32 studies were rated as being of excellent quality, 21 studies were rated as being of good quality, and 1 study was rated as being of fair quality; no study in the analysis was deemed to be of poor quality. Individual scoring is available in the online Supplementary Materials (https://osf.io/b28qd/).

\subsection{Main Models}

\subsubsection{Fat Mass}

The main model for all fat mass effects (55 across 29 clusters (median $=1$, range $=1-6$ effects per cluster)) revealed a trivial standardized point estimate with a high precision for the interval estimate $(-0.02(95 \% \mathrm{CI}=-0.07$ to 0.04$))$, with moderate heterogeneity 
$\left(Q_{(54)}=79.08, p=0.015, I^{2}=36 \%\right)$. Figure 3 presents all standardized effects and interval estimates for fat mass outcomes across studies in an ordered caterpillar plot.

Table 2. Descriptive characteristics.

\begin{tabular}{|c|c|}
\hline Characteristic & Number of Groups within Studies $=60$ \\
\hline Age (years) & $30(21,44)$ \\
\hline Unknown & 1 \\
\hline Sex (\% Male) & $54(32,100)$ \\
\hline $\operatorname{BMI}\left(\mathrm{kg} \cdot \mathrm{m}^{2}\right)$ & $28.3(25.4,30.5)$ \\
\hline Unknown & 7 \\
\hline Training Status & \\
\hline Recreationally Active & $1(1.7 \%)$ \\
\hline Trained & $1(1.7 \%)$ \\
\hline Untrained & $58(97 \%)$ \\
\hline \multicolumn{2}{|l|}{ Was Nutrition Controlled? } \\
\hline No & $31(52 \%)$ \\
\hline Yes & $29(48 \%)$ \\
\hline \multicolumn{2}{|l|}{ Included Caloric Deficit? } \\
\hline No & $57(95 \%)$ \\
\hline Yes & $3(5.0 \%)$ \\
\hline \multicolumn{2}{|l|}{ Include Resistance Training Intervention? } \\
\hline No & $59(98 \%)$ \\
\hline Yes & $1(1.7 \%)$ \\
\hline \multicolumn{2}{|l|}{ Were IT/MICT Work-Matched? } \\
\hline No & $34(57 \%)$ \\
\hline Yes & $25(42 \%)$ \\
\hline Yes, matched for time & $1(1.7 \%)$ \\
\hline Intervention Duration (weeks) & $12(8,12)$ \\
\hline \multicolumn{2}{|l|}{ IT Frequency (median days per week) } \\
\hline 2 & $8(13 \%)$ \\
\hline 3 & $44(73 \%)$ \\
\hline 3.5 & $2(3.3 \%)$ \\
\hline 4 & $3(5.0 \%)$ \\
\hline 4.5 & $2(3.3 \%)$ \\
\hline 5 & $1(1.7 \%)$ \\
\hline \multicolumn{2}{|l|}{ MICT Frequency (median days per week) } \\
\hline 2 & $8(13 \%)$ \\
\hline 3 & $38(63 \%)$ \\
\hline 3.5 & $2(3.3 \%)$ \\
\hline 4 & $3(5.0 \%)$ \\
\hline 4.5 & $2(3.3 \%)$ \\
\hline 5 & $7(12 \%)$ \\
\hline \multicolumn{2}{|l|}{ Was IT Performed as SIT or HIIT? } \\
\hline HIIT & $45(75 \%)$ \\
\hline SIT & $15(25 \%)$ \\
\hline IT Interval Number Performed & $5(4,10)$ \\
\hline Unknown & $\begin{array}{l}5 \\
5\end{array}$ \\
\hline IT Interval Duration (median s) & $60(30,180)$ \\
\hline IT Total Exercise Duration (min) & $9.4(3.4,16.0)$ \\
\hline MICT Session Duration (min) & $38(30,45)$ \\
\hline Unknown & 3 \\
\hline IT Adherence (\% Sessions) & $90(83,98)$ \\
\hline Unknown & 24 \\
\hline MICT Adherence (\% Sessions) & $90(84,97)$ \\
\hline Unknown & 25 \\
\hline \multicolumn{2}{|l|}{ IT Adverse Event Number } \\
\hline 0 & $12(63 \%)$ \\
\hline 1 & $2(11 \%)$ \\
\hline 2 & $2(11 \%)$ \\
\hline 3 & $1(5.3 \%)$ \\
\hline 4 & $1(5.3 \%)$ \\
\hline 5 & $1(5.3 \%)$ \\
\hline Unknown & 41 \\
\hline \multicolumn{2}{|l|}{ MICT Adverse Event Number } \\
\hline 0 & $10(67 \%)$ \\
\hline 1 & $3(20 \%)$ \\
\hline 2 & $2(13 \%)$ \\
\hline Unknown & 45 \\
\hline
\end{tabular}




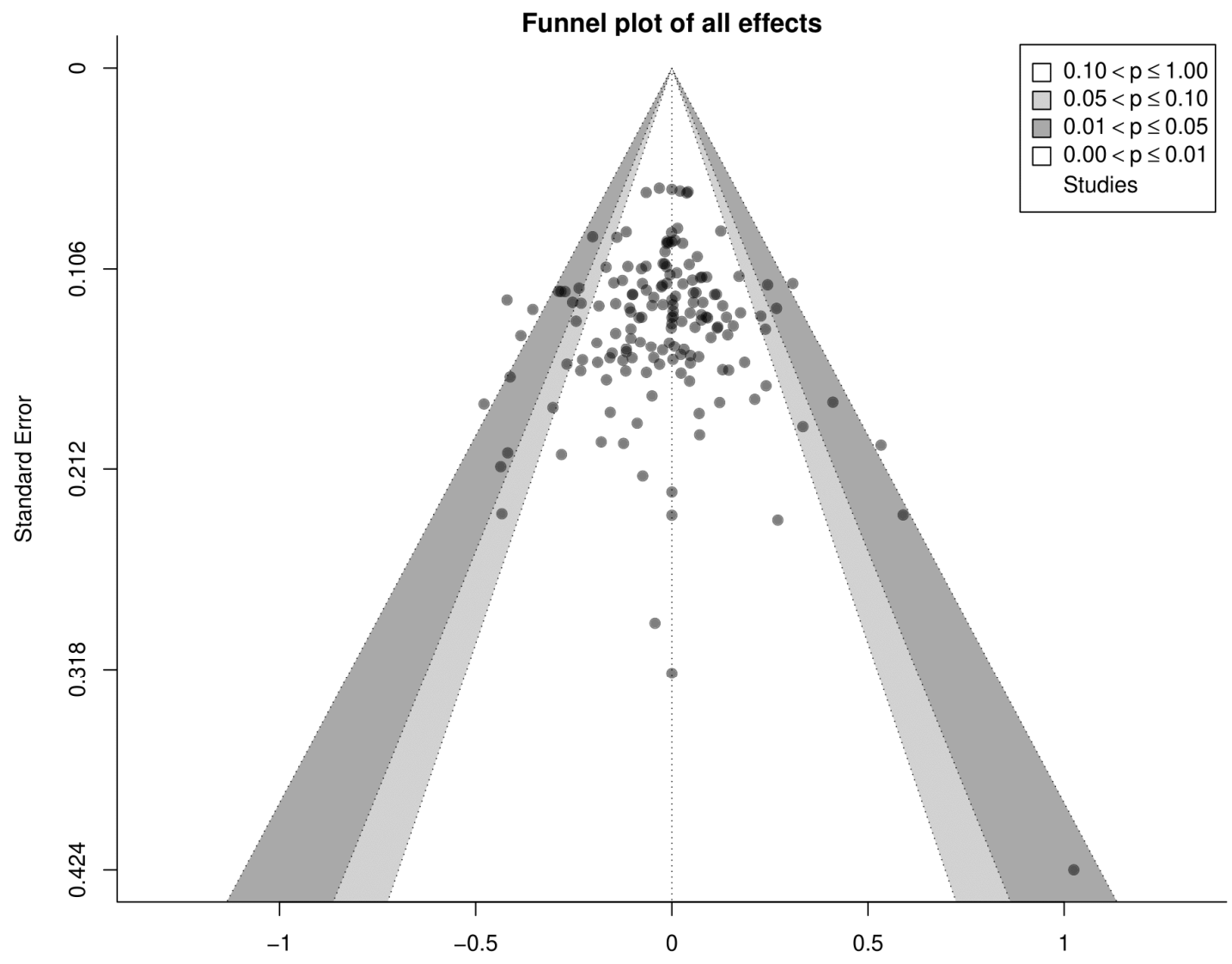

Figure 2. Contour enhanced funnel plot for all effects from the included studies.

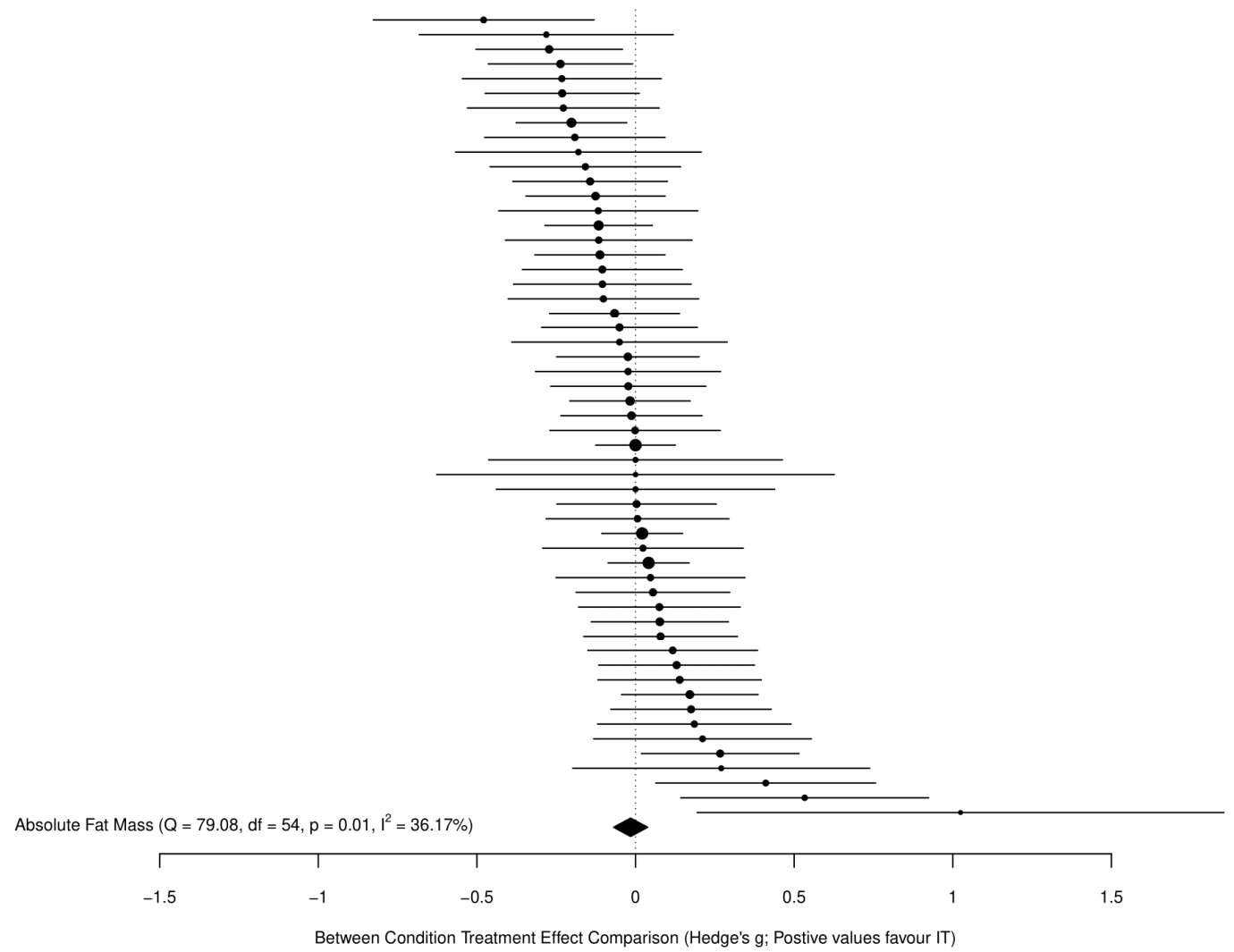

Figure 3. Standardized effects and interval estimates for fat mass outcomes across all studies in an ordered caterpillar plot. 


\subsubsection{Fat-Free Mass}

The main model for all FFM effects ( 34 across 27 clusters (median $=1$, range $=1-3$ effects per cluster)) revealed a trivial standardized point estimate with a high precision for the interval estimate $(-0.0004(95 \% \mathrm{CI}=-0.05$ to 0.05$))$, with negligible heterogeneity $\left(Q_{(33)}=37.77, p=0.26, I^{2}=16 \%\right)$. Figure 4 presents all standardized effects and interval estimates for FFM outcomes across studies in an ordered caterpillar plot.

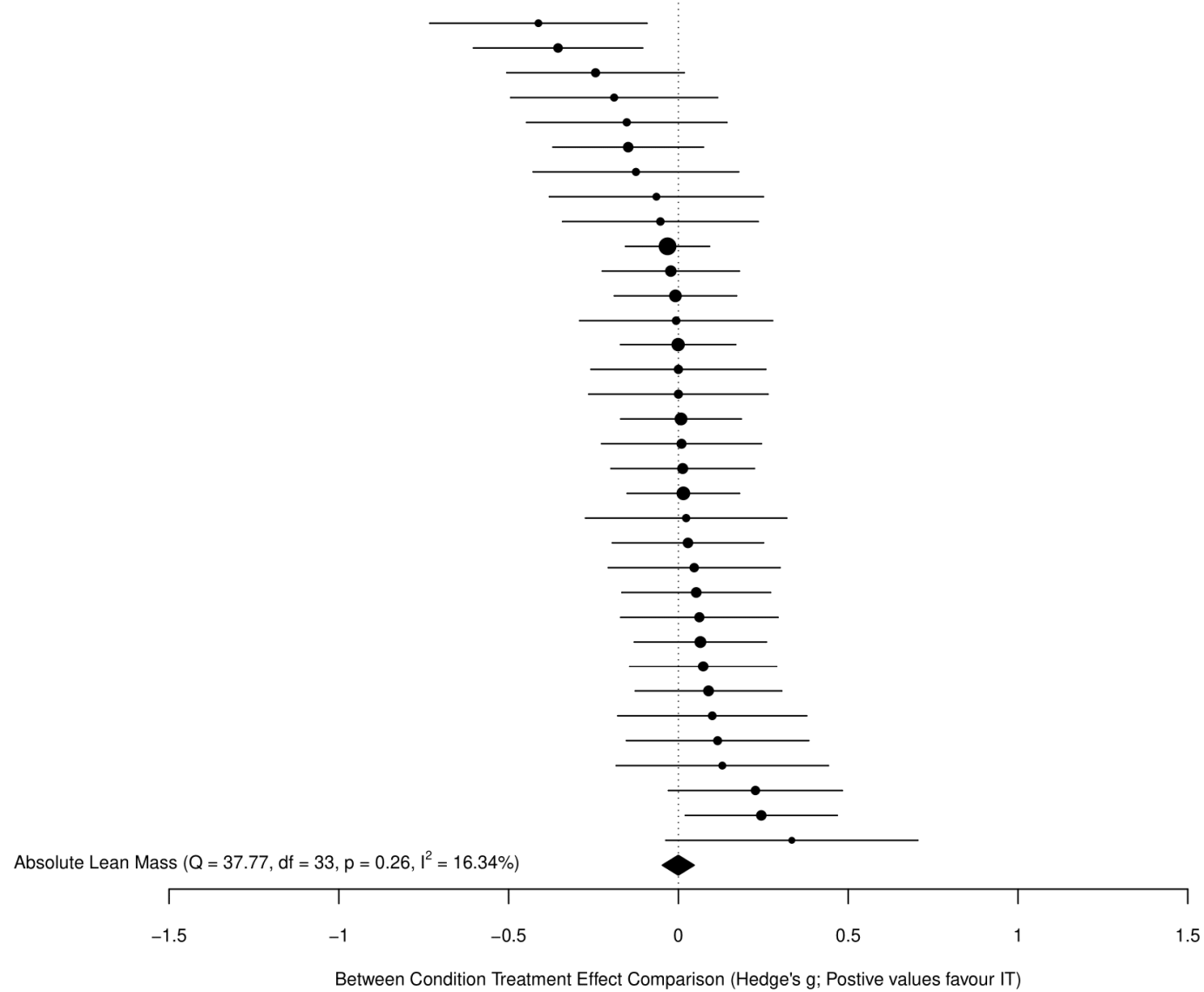

Figure 4. Standardized effects and interval estimates for FFM outcomes across studies in an ordered caterpillar plot.

\subsubsection{GRADE Summary of Findings for Main Outcomes}

For both fat mass and FFM there was a 'high' certainty of evidence with respect to the effects identified. It was deemed that there was no serious risk of bias, inconsistency, indirectness of evidence, or imprecision in estimates, nor were there other clear considerations impacting on certainty of evidence grading. The GRADE summary of findings table for our main outcomes is available in the Supplementary Materials (https://osf.io/pcyvx/).

\subsection{Secondary Analyses}

Between condition treatment effect models on both the raw effect scales, and using relative fat outcomes (relative lean models not run due to limited data), showed similar outcomes to the main models reported. Thus, for brevity, these are presented in the Supplementary Materials along with caterpillar plots (see folder "Outputs and Figures" > "Secondary Outcomes Outputs" > "Additional Between Condition Models" at https: //osf.io/6karz/).

\subsubsection{Within-Condition Treatment Effects}

All within-condition models are also available in the Supplementary Materials (see folder "Outputs and Figures" > "Secondary Outcomes Outputs" > "Within Condition 
Models" at https: / osf.io/6karz/) and here we report just the results for absolute fat and FFM outcomes on standardized and raw scales. In comparison to non-intervention control groups, the IT conditions resulted in small reductions in fat mass (Hedges' $\mathrm{g}=-0.22$ $(95 \% \mathrm{CI}=-0.36$ to -0.08$)$; kilograms $=-0.20(95 \% \mathrm{CI}=-0.34$ to -0.06$))$, and trivial increases in FFM (Hedges' $\mathrm{g}=0.13(95 \% \mathrm{CI}=0.04$ to 0.22$)$; and kilograms $=0.11(95 \% \mathrm{CI}=-0.04$ to 0.26$)$ ). The MICT conditions also produced small reductions in fat mass (Hedges' $\mathrm{g}=-0.20(95 \% \mathrm{CI}=-0.36$ to -0.04$) ;$ kilograms $=-0.25(95 \% \mathrm{CI}=-0.39$ to -0.11$))$, and trivial increases in FFM (Hedges' $\mathrm{g}=0.07(95 \% \mathrm{CI}=-0.01$ to 0.16$)$; kilograms $=0.07$ $(95 \% \mathrm{CI}=-0.02$ to 0.15$))$.

\subsubsection{Sub-Group and Meta-Regression Analyses}

Sub-group and meta-regression models were not run for absolute FFM standardized effects, given the negligible heterogeneity in the main model. When exploring subgroup and meta-regression models for absolute fat mass standardized effects, only two moderators-sex (proportion of males in sample; $\beta=0.0015(95 \% \mathrm{CI}=0.00$ to 0.0029$)$ ) and the number of intervals performed per training session by IT ( $\beta=-0.0032(95 \% \mathrm{CI}=-0.0052$ to -0.0013)) - appeared to have an effect, albeit this effect was relatively small for both covariates. Again, for brevity, all sub-group and meta-regression models are included in the Supplementary Materials (see folder "Outputs and Figures" > "Secondary Outcomes Outputs" > "Sub-group and Meta-regression Models" at https://osf.io/6karz/).

\subsubsection{Adherence, Dropouts and Adverse Events}

There was minimal difference in adherence or dropout proportions between conditions, which were relatively high and low, respectively. Adherence for IT was $89.1 \%$ $(95 \% \mathrm{CI}=85.2 \%$ to $92.1 \%)$ and for MICT was $89.2 \%(95 \% \mathrm{CI}=84.5 \%$ to $92.7 \%)$, and dropouts for IT were $16.1 \%(95 \% \mathrm{CI}=11.4 \%$ to $22.2 \%)$ and for MICT were $20.1 \%(95 \% \mathrm{CI}=12.3 \%$ to $33.1 \%$ ). Adverse events per 1000 person-sessions (i.e., the number of events per 1000 training sessions performed) were also relatively low with a minimal difference between conditions, with values of $1.15(95 \% \mathrm{CI}=0.31$ to 4.34$)$ and $1.07(95 \% \mathrm{CI}=0.51$ to 2.24$)$ for IT and MICT, respectively.

\subsubsection{Inter-Individual Response Variation}

There was no clear evidence of 'true' inter-individual variation in responses for either IT or MICT conditions. The difference in intercepts when compared with CON conditions were $-0.15(95 \% \mathrm{CI}=-0.35$ to 0.05$)$ and $-0.02(95 \% \mathrm{CI}=-0.22$ to 0.18$)$ for IT and MICT, respectively (see figure in Supplementary Materials: https:/ /osf.io/3mazj/).

\section{Discussion}

This is the most comprehensive meta-analysis to date comparing IT and MICT on changes of measures of fat mass and FFM. Furthermore, GRADE assessment suggests high certainty in the evidence presented. Our findings provide novel insights into the use of different training strategies to bring about changes in body composition. Below, we discuss the results and practical implications of our data for each outcome.

\subsection{Changes in Fat Mass}

It has been speculated that IT may confer superior fat loss benefits compared to MICT, primarily mediated via a greater excess post-exercise oxygen consumption (EPOC) [97]. However, the overall magnitude of additional energy expenditure attributed to EPOC during IT is modest [98], and thus is unlikely to be of practical meaningfulness from a fat loss standpoint. Other proposed benefits of IT on fat reduction include enhancements in appetite suppression, fat oxidation, and circulating catecholamines and lipolytic hormones [98]. Despite this mechanistic rationale, our results do not support a superiority of IT on reductions in fat mass. Analysis of standardized between-group treatment effects showed similar changes for IT and MICT with both absolute fat mass as our pri- 
mary outcome (Hedges' $\mathrm{g}=(-0.02(95 \% \mathrm{CI}=-0.07$ to 0.04$))$, and percentage body fat (Hedges' $\mathrm{g}=-0.04(95 \% \mathrm{CI}=-0.08$ to 0.01$)$ ). Raw absolute fat mass changes revealed a trivial point estimate of $-0.17 \mathrm{~kg}$ favoring MICT, although the interval estimate ranged from $-0.66 \mathrm{~kg}$ in favor of MICT to $0.31 \mathrm{~kg}$ in favor of IT. Comparison of raw relative (\%) fat mass changes in fat mass revealed a small point estimate of $-0.30 \%$ favoring MICT, but again, the interval estimate was imprecise, ranging from $-0.63 \%$ in favor of MICT to $0.04 \%$ in favor of IT. Taken as a whole, these findings suggest that changes in fat loss are not meaningfully influenced by patterns of intensity of effort and duration (i.e., IT vs MICT) during exercise.

When compared to non-exercising controls, IT and MICT produced small reductions in fat mass, with minimal differences between conditions. The raw absolute fat loss amounted to $-0.22 \mathrm{~kg}$ for IT and $-0.25 \mathrm{~kg}$ MICT, with standardized Hedges' $\mathrm{g}$ ES values of 0.22 and 0.20, respectively. Relative changes in fat mass for IT and MICT showed similarly small decreases vs controls, both on a raw $(0.30 \%$ and $0.25 \%$, respectively) and standardized ( 0.28 and 0.24 , respectively) basis. None of the studies that included control conditions combined exercise with dietary intervention (i.e., caloric deficit) and thus, collectively, these data suggest that exercise alone induces a small magnitude of fat loss regardless of the patterns of intensity of effort and duration, at least under the methods employed in current research. More extreme volumes of exercise may be necessary to induce meaningful changes, irrespective of the intensity of effort. The observed changes in fat mass $(\sim 0.2 \mathrm{~kg})$ in present studies and intervention examined are unlikely to be clinically or aesthetically meaningful in most populations. Indeed, these findings concur with earlier results from Keating et al. [8].

The lack of overall fat loss achieved in both IT and MICT can be attributed, at least in part, to the relatively low weekly exercise dose across studies (IT, median $=28 \mathrm{~min}$ duration (range $=3 \mathrm{~min}$ to $120 \mathrm{~min}$ ); $\mathrm{MICT}$, median $=120 \mathrm{~min}$ duration (range $=48 \mathrm{~min}$ to $250 \mathrm{~min}$ ), and perhaps is confounded by a corresponding increase in energy intake [99] and/or reduction in non-exercise activity thermogenesis [100]. Tightly controlled research in identical twins shows that prolonged daily aerobic-type exercise can induce marked reductions in fat mass under conditions of constant energy and nutrient intake [101]. However, the time commitment needed to achieve these results ( $100 \mathrm{~min} /$ day) is infeasible for the majority of the general public and is thus of limited practical relevance. Therefore, our findings underscore the importance of dietary prescription to facilitate weight loss; however, exercise may play an important supplementary role in the process [102].

In contrast to the recent meta-analysis from Sultana et al. [11], we did identify some moderate heterogeneity in our main model, leading us to explore possible moderators. For example, some evidence suggests that IT elicits greater reductions in abdominal adiposity compared to MICT [17]. Given the well-established association between android fat and cardiometabolic disease [103], such an outcome would potentially have major health implications if found to be true. However, our findings refute this contention, demonstrating similar changes in abdominal fat mass between conditions. Moreover, we found that relatively equal, albeit modest, fat loss occurred across the upper body, lower body and trunk regions regardless of condition, indicating that endurance-oriented exercise does not preferentially target specific fat deposits. Indeed, with the exception of sex and the number of intervals performed during IT training sessions, both of which also only had very trivial moderating effects, we did not identify any clear moderators of comparative treatment effects for fat mass.

\subsection{Changes in Fat-Free Mass}

Some researchers have proposed that the performance of aerobic exercise can elicit increases in skeletal muscle hypertrophy that are comparable to resistance exercise training [15]. However, a meta-analysis by Grgic et al. [104] refuted this contention, showing significantly greater hypertrophic adaptations from resistance training vs aerobic training, both at the whole-muscle and myofiber level. However, it should be noted that 
Grgic et al. [104] did not subanalyze the effects of endurance exercise intensity on hypertrophy outcomes. A recent review speculated that IT may provide sufficient stimulus to enhance muscle growth, particularly in middle-aged and older adults, as well as clinical populations [16]. Furthermore, some emerging evidence suggests that, although traditional resistance training and aerobic modality interventions may produce differing adaptations, when duration and intensity of effort are matched, similar strength and endurance adaptations may occur, although the impact on hypertrophy is less clear [105].

Our results suggest that endurance exercise intensity and duration may not mediate hypertrophic adaptations. Specifically, analysis of changes in FFM, both on an absolute and relative basis, demonstrated similar effects between IT and MICT. Between-condition treatment standardized effects for absolute changes in FFM were essentially zero $((-0.0004$ $(95 \% \mathrm{CI}=-0.05$ to 0.05$))$, and comparison of effects on the raw scale showed a small point estimate of $0.09 \mathrm{~kg}$ favoring IT, yet the interval estimate ranged from $-0.18 \mathrm{~kg}$ in favor of MICT, to $0.35 \mathrm{~kg}$ in favor of IT. There were limited data reporting relative changes in FFM, with only three studies directly comparing MICT vs IT. Pooling of these data revealed a moderate magnitude of effect $(-0.98 \%)$ favoring MICT. However, due to the lack of data, the confidence intervals around the point estimate were wide $(-3.39 \%$ to $1.43 \%)$, and Hedges' g values indicated a trivial standardized mean difference (0.17) with similarly wide interval estimates ( -0.69 to 0.35 ). From a practical standpoint, these findings collectively suggest there may not be a meaningful difference between MICT and IT on absolute changes in FFM.

Compared to non-exercising controls, our findings indicate trivial standardized effects for improvements in FFM for both conditions (IT, Hedges' $\mathrm{g}=0.13$ (95\% CI $=0.04$ to 0.22); MICT, Hedges' $\mathrm{g}=0.07(95 \% \mathrm{CI}=-0.01$ to 0.16$)$ ). IT showed absolute raw increases of $0.11 \mathrm{~kg}$ whereas MICT showed increases of 0.07 , although both the lower bounds of the interval estimates included zero and the upper bounds did not reach particularly meaningful values. These data collectively suggest that neither MICT nor IT meaningfully affect FFM under the methods employed across studies, and call into question the claim that endurance-based exercise is a viable interventional strategy for promoting muscle hypertrophy.

\subsection{Exercise Adherence and Dropouts}

Adherence was essentially identical between conditions, with both groups completing $\sim 90 \%$ of sessions; dropouts were also similar and relatively low at $\sim 13-17 \%$. It has been argued that the intensity of effort of exercise influences core affective response [106], and that this is predictive of future intentions and behavior in relation to exercise [107]. However, a recent systematic review suggests that affective response may only differ trivially between IT and MICT, and that enjoyment responses may demonstrate a small effect in favor of IT [108]. Despite varying speculative theories regarding the intensity of effort during exercise, and its impact on affect or enjoyment, and subsequent behaviors, the results here suggest that adherence to IT and MICT is largely similar and relatively high, at least over the duration of the studies and under the conditions in which the interventions were employed. Indeed, it should be noted that exercise sessions in the included studies were carried out with the aid of programming from the respective research teams and were generally performed under direct supervision. It is well-established that programming and supervision have positive effects on exercise adherence [109]. Thus, our findings in this regard cannot necessarily be extrapolated to self-directed exercise programs. Given the high interindividual variability observed in the psychological response to endurance exercise [110], it would seem that allowing for a choice of training intensity would likely help to improve long-term adherence. Future research should endeavor to test this hypothesis under ecologically valid conditions. 


\subsection{Adverse Events}

Of the studies reporting adverse events, there was essentially no difference between IT and MICT. On the surface, this would seem to suggest that both conditions are similarly safe in the populations studied. However, most studies failed to report incidences of adverse events. Furthermore, some studies lacked clarity as to whether there was a comprehensive attempt to record all possible adverse events associated with the training intervention. Thus, data on the topic is somewhat limited, precluding the ability to draw strong inferences regarding the safety between protocols.

A recent meta-analysis that examined the effects of supervised IT in patients with cardiovascular disease reported only five associated adverse cardiovascular events in approximately 17,000 training sessions: one major cardiovascular event, one minor cardiovascular, and three incidences of musculoskeletal issues. Although these findings appear to indicate that IT is generally safe, even in populations with non-communicable diseases and other health risks, results may be confounded by underreporting of adverse events in individual studies, and perhaps also by sampling bias for the types of individuals likely to participate in such studies. Researchers are thus encouraged to track and disclose the occurrence of such incidences in future studies on HIIT and MICT so that we can achieve a greater understanding of the risks associated with each strategy.

\subsection{Inter-Individual Response Variation}

Variance of treatment responses to IT and MICT has been relatively underexplored, despite numerous studies purporting that there may be inter-individual response variation to IT and MICT for a range of outcomes [19-21]. Indeed, some have argued that such variations may mask differences between IT and MICT for fat loss [22]. Evidence from the HERITAGE Family Study would genetically support this speculation, given that a putative dominant locus accounting for $31 \%$ of variance in fat mass changes was found [111]. However, we found no evidence of 'true' inter-individual variability in responses to either IT or MICT. This is in agreement with findings from a recent meta-analysis of aerobic exercise in overweight individuals and children and adolescents with obesity on fat loss [18]. Given our findings, and the relatively low heterogeneity across the main models for outcomes, the majority of apparent differences in study level results and apparent 'response heterogeneity' are likely attributable to sampling variance and random withinsubject variability.

\subsection{Limitations}

The present meta-analysis has several limitations that must be taken into account when attempting to draw practical inferences on the effects of IT vs MICT on measures of body composition. First and foremost, only three studies prescribed dietary energy restrictions for the interventional protocol. Thus, it is not clear whether one exercise strategy may be superior to another when combined with a nutritional intervention. Second, only one study supplemented the exercise intervention with a resistance training component. It is possible that differences in intensity and duration between IT and MICT protocols might alter responses when combined with resistance training. Although recent evidence questions whether there is an interference effect from concurrent training, at least for hypertrophy [112], the specific roles of endurance exercise intensity and duration upon fat mass under these conditions have yet to be elucidated. Third, very few studies involved trained athletes, and the vast majority of subjects would be considered to be overweight/obese. Thus, it remains to be determined how differences in endurance exercise intensity and duration may affect body composition outcomes in lean and athletic populations. Moreover, the majority of included studies examined outcomes in younger to middle-aged adults, limiting our ability to draw conclusions about the effects of IT and MICT on older populations. Fourth, although we were able to separate studies that had included control groups for the purpose of a 'within-condition' analysis of the true treatment effects for IT and MICT, in addition to exploration of interindividual response variability, these were 
secondary exploratory analyses. Our search strategy and inclusion were not optimized to identify all studies that included either IT or MICT and a non-training CON condition. However, our estimates for within-group IT effects were not dissimilar to those reported by Sultana et al. [11] for IT vs CON, who did include studies with either an MICT or a nontraining CON condition. Finally, our analysis is specific to body composition changes and does not take into account the other potential effects of the different interventional exercise strategies. Some evidence indicates that higher intensities of exercise may confer superior health-related benefits such as improvements in glucose control, blood pressure, vascular function, and cardiorespiratory fitness [113]. Thus, the use of a given endurance exercise strategy should consider individual goals in combination with abilities and preferences.

\section{Conclusions}

Our findings provide compelling evidence that the patterns of intensity of effort and duration during endurance exercise has minimal influence on longitudinal changes in fat mass and FFM. From a practical standpoint, this implies that individuals can choose the intensity of effort and duration combination (i.e., IT or MICT) that best suits their needs and lifestyle. As a general rule, there is an efficiency/effort tradeoff along the intensity of effort spectrum, whereby IT requires less time but more effort than MICT to promote alterations in body composition. Given that exercise adherence is of paramount concern, personal preference should thus guide prescription.

Our findings also indicate that structured exercise only has minor effects on fat loss regardless of intensity of effort and duration when performed at relatively modest doses; the amount of exercise required to achieve practically meaningful changes in this outcome seems to be unrealistic for most individuals. It is much easier to create an energy deficit from dietary restriction, which, therefore, should be the focus of weight loss interventions. However, exercise may help to preserve FFM and functional performance during periods of energy restriction [114], as well as facilitate sustenance of weight loss in combination with a dietary intervention [115]. Thus, it should be considered an important adjunct to nutritional approaches for those who endeavor to alter their body composition.

Supplementary Materials: The following are available online at https:/ /osf.io/6karz/; https:/ osf. io/dumq8/; https://osf.io/b28qd/; https:/ /osf.io/pcyvx/; https:/ /osf.io/3mazj/.

Author Contributions: B.J.S. conceived of the study, designed the methods, and conducted the quality assessment; J.S. assisted in the methods design and carried out statistical analyses; J.G. conducted the quality assessment; D.P., A.R. and M.C.-M. carried out the search; D.V.E., H.Z. and B.M. coded the studies; all authors were meaningfully involved in interpreting data, and drafting and critically revising the manuscript for intellectually important content. All authors have read and agreed to the published version of the manuscript.

Funding: No funding was received for this manuscript.

Institutional Review Board Statement: Not applicable.

Informed Consent Statement: Not applicable.

Data Availability Statement: All data, code, materials and supplementary analyses are openly available on the Open Science Framework page at: https://osf.io/6karz/.

Conflicts of Interest: The authors report no conflicts of interest with this manuscript.

\section{References}

1. Genton, L.; Graf, C.E.; Karsegard, V.L.; Kyle, U.G.; Pichard, C. Low fat-free mass as a marker of mortality in community-dwelling healthy elderly subjects. Age Ageing 2013, 42, 33-39. [CrossRef] [PubMed]

2. Lima, R.M.; Bezerra, L.M.; Rabelo, H.T.; Silva, M.A.; Silva, A.J.; Bottaro, M.; de Oliveira, R.J. Fat-free mass, strength, and sarcopenia are related to bone mineral density in older women. J. Clin. Densitom. 2009, 12, 35-41. [CrossRef]

3. Haykowsky, M.J.; Brubaker, P.H.; Morgan, T.M.; Kritchevsky, S.; Eggebeen, J.; Kitzman, D.W. Impaired aerobic capacity and physical functional performance in older heart failure patients with preserved ejection fraction: Role of lean body mass. J. Gerontol. A Biol. Sci. Med. Sci. 2013, 68, 968-975. [CrossRef] [PubMed] 
4. Hydren, J.R.; Borges, A.S.; Sharp, M.A. Systematic review and meta-analysis of predictors of military task performance: Maximal lift capacity. J. Strength Cond. Res. 2017, 31, 1142-1164. [CrossRef]

5. Zamboni, M.; Mazzali, G.; Fantin, F.; Rossi, A.; Di Francesco, V. Sarcopenic obesity: A new category of obesity in the elderly. Nutr. Metab. Cardiovasc. Dis. 2008, 18, 388-395. [CrossRef]

6. Stoner, L.; Rowlands, D.; Morrison, A.; Credeur, D.; Hamlin, M.; Gaffney, K.; Lambrick, D.; Matheson, A. Efficacy of exercise intervention for weight loss in overweight and obese adolescents: Meta-analysis and implications. Sports Med. 2016, 46, 1737-1751. [CrossRef]

7. Miller, C.T.; Fraser, S.F.; Levinger, I.; Straznicky, N.E.; Dixon, J.B.; Reynolds, J.; Selig, S.E. The effects of exercise training in addition to energy restriction on functional capacities and body composition in obese adults during weight loss: A systematic review. PLOS ONE 2013, 8, e81692.

8. Keating, S.E.; Johnson, N.A.; Mielke, G.I.; Coombes, J.S. A systematic review and meta-analysis of interval training versus moderate-intensity continuous training on body adiposity. Obes. Rev. 2017, 18, 943-964. [CrossRef]

9. Viana, R.B.; Naves, J.P.A.; Coswig, V.S.; de Lira, C.A.B.; Steele, J.; Fisher, J.P.; Gentil, P. Is interval training the magic bullet for fat loss? A systematic review and meta-analysis comparing moderate-intensity continuous training with high-intensity interval training (HIIT). Br. J. Sports Med. 2019, 53, 655-664. [CrossRef]

10. Hollings, M.; Coombes, J.; Mavros, Y.; Keating, S.; Fiatarone-Singh, M. Expression of concern: Is interval training the magic bullet for fat loss? A systematic review and meta-analysis comparing moderate-intensity continuous training with high-intensity training (HIIT). Br. J. Sports Med. 2019. [CrossRef]

11. Sultana, R.N.; Sabag, A.; Keating, S.E.; Johnson, N.A. The effect of low-volume high-intensity interval training on body composition and cardiorespiratory fitness: A systematic review and meta-analysis. Sports Med. 2019, 49, 1687-1721. [CrossRef] [PubMed]

12. Morris, B. Estimating effect sizes from pretest-posttest-control group designs. Organ. Res. Methods 2008, 11, 364-386. [CrossRef]

13. Moeyaert, M.; Ugille, M.; Beretvas, S.N.; Ferron, J.; Bunuan, R.; Van den Noortgate, W. Methods for dealing with multiple outcomes in meta-analysis: A comparison between averaging effect sizes, robust variance estimation and multilevel meta-analysis. Int. J. Soc.Res. Methodol. 2017, 20, 559-572. [CrossRef]

14. Higgins, J.P.T.; Thomas, J.; Chandler, J.; Cumpston, M.; Li, T.; Page, M.J.; Welch, V.A. Cochrane Handbook for Systematic Reviews of Interventions, Version 6.2 ed.; Cochrane: London, UK, 2021; Available online: www.training.cochrane.org/handbook (accessed on 6 November 2021).

15. Konopka, A.R.; Harber, M.P. Skeletal muscle hypertrophy after aerobic exercise training. Exerc. Sport Sci. Rev. 2014, $42,53$. [CrossRef]

16. Callahan, M.J.; Parr, E.B.; Hawley, J.A.; Camera, D.M. Can high-intensity interval training promote skeletal muscle anabolism? Sports Med. 2021, 51, 405-421. [CrossRef]

17. Maillard, F.; Rousset, S.; Pereira, B.; Boirie, Y.; Duclos, M.; Boisseau, N. High-intensity interval training is more effective than moderate-intensity continuous training in reducing abdominal fat mass in postmenopausal women with type 2 diabetes: A randomized crossover study. Diabetes Metab. 2018, 44, 516-517. [CrossRef] [PubMed]

18. Kelley, G.A.; Kelley, K.S.; Pate, R.R. Are there inter-individual differences in fat mass and percent body fat as a result of aerobic exercise training in overweight and obese children and adolescents? A meta-analytic perspective. Child. Obes. 2020, 16, 301-306. [CrossRef]

19. Düking, P.; Holmberg, H.C.; Kunz, P.; Leppich, R.; Sperlich, B. Intra-individual physiological response of recreational runners to different training mesocycles: A randomized cross-over study. Eur. J. Appl. Physiol. 2020, 120, 2705-2713. [CrossRef]

20. Bonet, J.B.; Magalhães, J.; Viscor, G.; Pagès, T.; Ventura, J.L.; Torrella, J.R.; Javierre, C. Inter-individual different responses to continuous and interval training in recreational middle-aged women runners. Front. Physiol. 2020, 11, 579835. [CrossRef]

21. Schulhauser, K.T.; Bonafiglia, J.T.; McKie, G.L.; McCarthy, S.F.; Islam, H.; Townsend, L.K.; Grisebach, D.; Todd, C.; Gurd, B.J.; Hazell, T. Individual patterns of response to traditional and modified sprint interval training. J. Sports Sci. 2021, 39, $1077-1087$. [CrossRef]

22. Tong, T.K.; Zhang, H.; Shi, H.; Liu, Y.; Ai, J.; Nie, J.; Kong, Z. Comparing time efficiency of sprint vs. high-intensity interval training in reducing abdominal visceral fat in obese young women: A randomized, controlled trial. Front. Physiol. 2018, 9, 1048. [CrossRef]

23. Atkinson, G.; Williamson, P.; Batterham, A.M. Issues in the determination of 'responders' and 'non-responders' in physiological research. Exp. Physiol. 2019, 104, 1215-1225. [CrossRef]

24. Senior, A.M.; Viechtbauer, W.; Nakagawa, S. Revisiting and expanding the meta-analysis of variation: The log coefficient of variation ratio. Res. Synth. Methods 2020, 11, 553-567. [CrossRef]

25. Moher, D.; Liberati, A.; Tetzlaff, J.; Altman, D.G.; PRISMA Group. Preferred reporting items for systematic reviews and meta-analyses: The PRISMA statement. PLoS Med. 2009, 6, e1000097. [CrossRef]

26. Greenhalgh, T.; Peacock, R. Effectiveness and efficiency of search methods in systematic reviews of complex evidence: Audit of primary sources. BMJ 2005, 331, 1064-1065. [CrossRef]

27. Cooper, H.; Hedges, L.; Valentine, J. The Handbook of Research Synthesis and Meta-Analysis, 2nd ed.; Russell Sage Foundation: New York, NY, USA, 2009. 
28. Elkins, M.R.; Herbert, R.D.; Moseley, A.M.; Sherrington, C.; Maher, C. Rating the quality of trials in systematic reviews of physical therapy interventions. Cardiopulm. Phys.Ther. J. 2010, 21, 20-26. [CrossRef] [PubMed]

29. Moseley, A.M.; Herbert, R.D.; Sherrington, C.; Maher, C.G. Evidence for physiotherapy practice: A survey of the Physiotherapy Evidence Database (PEDro). Aust. J. Physiother. 2002, 48, 43-49. [CrossRef]

30. Kummel, J.; Kramer, A.; Giboin, L.S.; Gruber, M. Specificity of balance training in healthy individuals: A systematic review and meta-analysis. Sports Med. 2016, 46, 1261-1271. [CrossRef] [PubMed]

31. Schünemann, H.; Brożek, J.; Guyatt, G.; Oxman, A.; The GRADE Working Group. GRADE Handbook for Grading Quality of Evidence and Strength of Recommendations. 2013. Available online: https://gdt.gradepro.org/app/handbook/handbook.html (accessed on 11 July 2021).

32. GRADEpro Guideline Development Tool; McMaster University: Hamilton, ON, Canada, 2020; Available online: https://gradepro. org/ (accessed on 30 June 2021).

33. Viechtbauer, W. Conducting meta-analysis in R with metafor package. J. Stat. Software 2010, 36, 1-48. [CrossRef]

34. Cohen, J. Statistical Power Analysis for the Behavioral Sciences, 2nd ed.; Lawrence Erlbaum: Hillsdale, NJ, USA, 1988.

35. Amrhein, V.; Greenland, S.; McShane, B. Scientists rise up against statistical significance. Nature 2019, 567, 305-307. [CrossRef] [PubMed]

36. McShane, B.B.; Gal, D.; Gelman, A.; Robert, C.; Tackett, J.L. Abandon statistical significance. Am. Stat. 2019, 73 (Suppl. 1), $235-245$. [CrossRef]

37. Higgins, J.P.; Thompson, S.G.; Deeks, J.J.; Altman, D.G. Measuring inconsistency in meta-analyses. BMJ 2003, 327, 557-560. [CrossRef]

38. Higgins, J.P.; Altman, D.G.; Gøtzsche, P.C.; Jüni, P.; Moher, D.; Oxman, A.D.; Savović, J.; Schulz, K.F.; Weeks, L.; Sterne, J.A. The Cochrane Collaboration's tool for assessing risk of bias in randomised trials. BMJ 2011, 343, d5928. [CrossRef]

39. Hecksteden, A.; Kraushaar, J.; Scharhag-Rosenberger, F.; Theisen, D.; Senn, S.; Meyer, T. Individual response to exercise training-A statistical perspective. J. Appl. Physiol. 2015, 118, 1450-1459. [CrossRef]

40. Nakagawa, S.; Poulin, R.; Mengersen, K.; Reinhold, K.; Engqvist, L.; Lagisz, M.; Senior, A.M. Meta-analysis of variation: Ecological and evolutionary applications and beyond. Methods Ecol. Evol. 2015, 6, 143-152. [CrossRef]

41. Connolly, L.J.; Nordsborg, N.B.; Nyberg, M.; Weihe, P.; Krustrup, P.; Mohr, M. Low-volume high-intensity swim training is superior to high-volume low-intensity training in relation to insulin sensitivity and glucose control in inactive middle-aged women. Eur. J. Appl. Physiol. 2016, 116, 1889-1897. [CrossRef] [PubMed]

42. Safarimosavi, S.; Mohebbi, H.; Rohani, H. High-intensity interval vs. continuous endurance training: Preventive effects on hormonal changes and physiological adaptations in prediabetes patients. J. Strength Cond. Res. 2021, 35, 731-738. [CrossRef] [PubMed]

43. De Araujo, A.C.C.; Roschel, H.; Picanço, A.R.; do Prado, D.M.; Villares, S.M.; de Sá Pinto, A.L.; Gualano, B. Similar health benefits of endurance and high-intensity interval training in obese children. PLoS ONE 2012, 7, e42747. [CrossRef]

44. Boer, P.H.; Meeus, M.; Terblanche, E.; Rombaut, L.; Wandele, I.D.; Hermans, L.; Gysel, T.; Ruige, J.; Calders, P. The influence of sprint interval training on body composition, physical and metabolic fitness in adolescents and young adults with intellectual disability: A randomized controlled trial. Clin. Rehabil. 2014, 28, 221-231. [CrossRef] [PubMed]

45. Boer, P.H.; Moss, S.J. Effect of continuous aerobic vs. interval training on selected anthropometrical, physiological and functional parameters of adults with Down syndrome. J. Intellect. Disabil. Res. 2016, 60, 322-334. [CrossRef]

46. Buchan, D.S.; Ollis, S.; Young, J.D.; Thomas, N.E.; Cooper, S.M.; Tong, T.K.; Nie, J.; Malina, R.M.; Baker, J.S. The effects of time and intensity of exercise on novel and established markers of CVD in adolescent youth. Am. J. Hum. Biol. 2011, 23, 517-526. [CrossRef] [PubMed]

47. Camacho-Cardenosa, A.; Brazo-Sayavera, J.; Camacho-Cardenosa, M.; Marcos-Serrano, M.; Timón, R.; Olcina, G. Effects of high intensity interval training on fat mass parameters in adolescents. Rev. Esp. Salud Publica 2016, 90, e1-e9. [PubMed]

48. Cheema, B.S.; Davies, T.B.; Stewart, M.; Papalia, S.; Atlantis, E. The feasibility and effectiveness of high-intensity boxing training versus moderate-intensity brisk walking in adults with abdominal obesity: A pilot study. BMC Sports Sci. Med. Rehabil. 2015, 7, 3 . [CrossRef]

49. Cocks, M.; Shaw, C.S.; Shepherd, S.O.; Fisher, J.P.; Ranasinghe, A.; Barker, T.A.; Wagenmakers, A.J. Sprint interval and moderateintensity continuous training have equal benefits on aerobic capacity, insulin sensitivity, muscle capillarisation and endothelial eNOS/NAD(P)Hoxidase protein ratio in obese men. J. Physiol. 2016, 594, 2307-2321. [CrossRef]

50. Devin, J.L.; Sax, A.T.; Hughes, G.I.; Jenkins, D.G.; Aitken, J.F.; Chambers, S.K.; Dunn, J.C.; Bolam, K.A.; Skinner, T.L. The influence of high-intensity compared with moderate-intensity exercise training on cardiorespiratory fitness and body composition in colorectal cancer survivors: A randomised controlled trial. J. Cancer Surviv. 2016, 10, 467-479. [CrossRef] [PubMed]

51. Dias, K.A.; Ingul, C.B.; Tjønna, A.E.; Keating, S.E.; Gomersall, S.R.; Follestad, T.; Hosseini, M.S.; Hollekim-Strand, S.M.; Ro, T.B.; Haram, M.; et al. Effect of high-intensity interval training on fitness, fat mass and cardiometabolic biomarkers in children with obesity: A randomised controlled trial. Sports Med. 2018, 48, 733-746. [CrossRef]

52. Earnest, C.P.; Lupo, M.; Thibodaux, J.; Hollier, C.; Butitta, B.; Lejeune, E.; Johannsen, N.M.; Gibala, M.J.; Church, T.S. Interval training in men at risk for insulin resistance. Int. J. Sports Med. 2013, 34, 355-363. [CrossRef]

53. Eimarieskandari, R.; Zilaeibouri, S.; Zilaeibouri, M.; Ahangarpour, A. Comparing two modes of exercise training with different intensity on body composition in obese young girls. Ovidius Univ. Ann. Ser. Phys. Educ. Sport Mov. Health 2012, 12, 473-478. 
54. Elmer, D.J.; Laird, R.H.; Barberio, M.D.; Pascoe, D.D. Inflammatory, lipid, and body composition responses to interval training or moderate aerobic training. Eur. J. Appl. Physiol. 2016, 116, 601-609. [CrossRef]

55. Fisher, G.; Brown, A.W.; Brown, M.M.B.; Alcorn, A.; Noles, C.; Winwood, L.; Resuehr, H.; George, B.; Jeansonne, M.M.; Allison, D.B. High intensity interval- vs moderate intensity- training for improving cardiometabolic health in overweight or obese males: A randomized controlled trial. PLoS ONE 2015, 10, e0138853. [CrossRef]

56. Galedari, M.; Azarbayjani, M.A.; Peeri, M. Effects of type of exercise along with caloric restriction on plasma apelin 36 and HOMA-IR in overweight men. Sci. Sports 2017, 32, e137-e145. [CrossRef]

57. Gillen, J.B.; Martin, B.J.; MacInnis, M.J.; Skelly, L.E.; Tarnopolsky, M.A.; Gibala, M.J. Twelve weeks of sprint interval training improves indices of cardiometabolic health similar to traditional endurance training despite a five-fold lower exercise volume and time commitment. PLoS ONE 2016, 11, e0154075.

58. Gripp, F.; Nava, R.C.; Cassilhas, R.C.; Esteves, E.A.; Magalhães, C.O.D.; Dias-Peixoto, M.F.; de Castro Magalhães, F.; Amorim, F.T. HIIT is superior than MICT on cardiometabolic health during training and detraining. Eur. J. Appl. Physiol. 2021, 121, 159-172. [CrossRef]

59. Higgins, S.; Fedewa, M.V.; Hathaway, E.D.; Schmidt, M.D.; Evans, E.M. Sprint interval and moderate-intensity cycling training differentially affect adiposity and aerobic capacity in overweight young-adult women. Appl. Physiol. Nutr. Metab. 2016, 41, 1177-1183. [CrossRef]

60. Hwang, C.L.; Yoo, J.K.; Kim, H.K.; Hwang, M.H.; Handberg, E.M.; Petersen, J.W.; Christou, D.D. Novel all-extremity highintensity interval training improves aerobic fitness, cardiac function and insulin resistance in healthy older adults. Exp. Gerontol. 2016, 82, 112-119. [CrossRef]

61. Keating, S.E.; Machan, E.A.; O'Connor, H.T.; Gerofi, J.A.; Sainsbury, A.; Caterson, I.D.; Johnson, N.A. Continuous exercise but not high intensity interval training improves fat distribution in overweight adults. J. Obes. 2014, 2014, 834865. [CrossRef]

62. Koubaa, A. Effect of intermittent and continuous training on body composition cardiorespiratory fitness and lipid profile in obese adolescents. IOSR J. Pharm. 2013, 3, 31-37. [CrossRef]

63. Lunt, H.; Draper, N.; Marshall, H.C.; Logan, F.J.; Hamlin, M.J.; Shearman, J.P.; Cotter, J.D.; Kimber, N.E.; Blackwell, G.; Frampton, C.M. High intensity interval training in a real world setting: A randomized controlled feasibility study in overweight inactive adults, measuring change in maximal oxygen uptake. PLoS ONE 2014, 9, e83256.

64. Macpherson, R.E.; Hazell, T.J.; Olver, T.D.; Paterson, D.H.; Lemon, P.W. Run sprint interval training improves aerobic performance but not maximal cardiac output. Med. Sci. Sports Exerc. 2011, 43, 115-122. [CrossRef]

65. Mäder, U.; Roth, P.; Furrer, R.; Brêchet, J.P.; Boutellier, U. Influence of continuous and discontinuous training protocols on subcutaneous adipose tissue and plasma substrates. Int. J. Sports Med. 2001, 22, 344-349. [CrossRef] [PubMed]

66. Magalhães, J.P.; Santos, D.A.; Correia, I.R.; Hetherington-Rauth, M.; Ribeiro, R.; Raposo, J.F.; Matos, A.; Bicho, M.D.; Sardinha, L.B. Impact of combined training with different exercise intensities on inflammatory and lipid markers in type 2 diabetes: A secondary analysis from a 1-year randomized controlled trial. Cardiovasc. Diabetol. 2020, 19, 169. [CrossRef]

67. Maillard, F.; Rousset, S.; Pereira, B.; Traore, A.; Del Amaze, P.d.P.; Boirie, Y.; Duclos, M.; Boisseau, N. High-intensity interval training reduces abdominal fat mass in postmenopausal women with type 2 diabetes. Diabetes Metab. 2016, 42, 433-441. [CrossRef] [PubMed]

68. Martínez, S.R.; Ríos, L.J.C.; Tamayo, I.M.; Almeida, L.G.; López-Gomez, M.A.; Jara, C.C. An after-school, high-intensity, interval physical activity programme improves health-related fitness in children. Mot. Rev. Educ. Fis. 2016, 22, 359-367. [CrossRef]

69. Martins, C.; Kazakova, I.; Ludviksen, M.; Mehus, I.; Wisloff, U.; Kulseng, B.; Morgan, L.; King, N. High-intensity interval training and isocaloric moderate-intensity continuous training result in similar improvements in body composition and fitness in obese individuals. Int. J. Sport Nutr. Exerc. Metab. 2016, 26, 197-204. [CrossRef]

70. Matsuo, T.; Saotome, K.; Seino, S.; Eto, M.; Shimojo, N.; Matsushita, A.; Iemitsu, M.; Ohshima, H.; Tanaka, K.; Mukai, C. Low-volume, high-intensity, aerobic interval exercise for sedentary adults: $\mathrm{VO}_{2} \mathrm{max}$, cardiac mass, and heart rate recovery. Eur. J. Appl. Physiol. 2014, 114, 1963-1972. [CrossRef] [PubMed]

71. Matsuo, T.; So, R.; Shimojo, N.; Tanaka, K. Effect of aerobic exercise training followed by a low-calorie diet on metabolic syndrome risk factors in men. Nutr Metab Cardiovasc. Dis. 2015, 25, 832-838. [CrossRef] [PubMed]

72. Mohr, M.; Nordsborg, N.B.; Lindenskov, A.; Steinholm, H.; Nielsen, H.P.; Mortensen, J.; Weihe, P.; Krustrup, P. High-intensity intermittent swimming improves cardiovascular health status for women with mild hypertension. BioMed Res. Int. 2014, 2014, 728289. [CrossRef]

73. Moreira, M.M.; Souza, H.P.; Schwingel, P.A.; Sá, C.K.; Zoppi, C.C. Effects of aerobic and anaerobic exercise on cardiac risk variables in overweight adults. Arq. Bras. Cardiol. 2008, 91, 219-226. [CrossRef]

74. Morrissey, C.; Montero, D.; Raverdy, C.; Masson, D.; Amiot, M.J.; Vinet, A. Effects of exercise intensity on microvascular function in obese adolescents. Int. J. Sports Med. 2018, 39, 450-455. [CrossRef]

75. Murphy, A.; Kist, C.; Gier, A.J.; Edwards, N.M.; Gao, Z.; Siegel, R.M. The feasibility of high-intensity interval exercise in obese adolescents. Clin. Pediatr. 2015, 54, 87-90. [CrossRef]

76. Nalcakan, G.R. The effects of sprint interval vs. continuous endurance training on physiological and metabolic adaptations in young healthy adults. J. Hum. Kinet. 2014, 44, 97-109. [CrossRef] [PubMed] 
77. Nybo, L.; Sundstrup, E.; Jakobsen, M.D.; Mohr, M.; Hornstrup, T.; Simonsen, L.; Bülow, J.; Randers, M.B.; Nielsen, J.J.; Aagaard, P.; et al. High-intensity training versus traditional exercise interventions for promoting health. Med. Sci. Sports Exerc. 2010, 42 1951-1958. [CrossRef] [PubMed]

78. Oh, S.; So, R.; Shida, T.; Matsuo, T.; Kim, B.; Akiyama, K.; Isobe, T.; Okamoto, Y.; Tanaka, K.; Shoda, J. High-intensity aerobic exercise improves both hepatic fat content and stiffness in sedentary obese men with nonalcoholic fatty liver disease. Sci. Rep. 2017, 7, 43029. [CrossRef] [PubMed]

79. Panissa, V.; Alves, E.; Salermo, G.; Franchini, E.; Takito, M. Can short-term high-intensity intermittent training reduce adiposity? Sports Sci. Health 2016, 12, 99-104. [CrossRef]

80. Pasetti, S.R.; Gonçalves, A.; Padovani, C.R. Continuous training versus interval training in deep water running: Health effects for obese women. Rev. Andal. Med. Deport. 2012, 5, 3-7. [CrossRef]

81. Ramírez-Vélez, R.; Tordecilla-Sanders, A.; Téllez, T.L.A.; Camelo-Prieto, D.; Hernández-Quiñonez, P.A.; Correa-Bautista, J.E.; Garcia-Hermoso, A.; Ramirez-Campillo, R.; Izquierdo, M. Similar cardiometabolic effects of high- and moderate-intensity training among apparently healthy inactive adults: A randomized clinical trial. J. Transl. Med. 2017, 15, 118. [CrossRef]

82. Ramos, J.S.; Dalleck, L.C.; Borrani, F.; Mallard, A.R.; Clark, B.; Keating, S.E.; Fassett, R.G.; Coombes, J.S. The effect of different volumes of high-intensity interval training on proinsulin in participants with the metabolic syndrome: A randomised trial. Diabetologia 2016, 59, 2308-2320. [CrossRef]

83. Reljic, D.; Wittmann, F.; Fischer, J.E. Effects of low-volume high-intensity interval training in a community setting: A pilot study. Eur. J. Appl. Physiol. 2018, 118, 1153-1167. [CrossRef]

84. Sasaki, H.; Morishima, T.; Hasegawa, Y.; Mori, A.; Ijichi, T.; Kurihara, T.; Goto, K. 4 weeks of high-intensity interval training does not alter the exercise-induced growth hormone response in sedentary men. Springerplus 2014, 3, 336. [CrossRef]

85. Schjerve, I.E.; Tyldum, G.A.; Tjønna, A.E.; Stølen, T.; Loennechen, J.P.; Hansen, H.E.; Haram, P.M.; Heinrich, G.; Bye, A.; Najjar, S.M.; et al. Both aerobic endurance and strength training programmes improve cardiovascular health in obese adults. Clin. Sci. 2008, 115, 283-293. [CrossRef]

86. Shepherd, S.O.; Cocks, M.; Tipton, K.D.; Ranasinghe, A.M.; Barker, T.A.; Burniston, J.G.; Wagenmakers, A.J.; Shaw, C.S. Sprint interval and traditional endurance training increase net intramuscular triglyceride breakdown and expression of perilipin 2 and 5. J. Physiol. 2013, 591, 657-675. [CrossRef] [PubMed]

87. Shepherd, S.O.; Wilson, O.J.; Taylor, A.S.; Thøgersen-Ntoumani, C.; Adlan, A.M.; Wagenmakers, A.J.; Shaw, C.S. Low-volume high-intensity interval training in a gym setting improves cardio-metabolic and psychological health. PLoS ONE 2015, 10, e0139056.

88. Shing, C.M.; Webb, J.J.; Driller, M.W.; Williams, A.D.; Fell, J.W. Circulating adiponectin concentration and body composition are altered in response to high-intensity interval training. J. Strength Cond. Res. 2013, 27, 2213-2218. [CrossRef]

89. Sijie, T.; Hainai, Y.; Fengying, Y.; Jianxiong, W. High intensity interval exercise training in overweight young women. J. Sports Med. Phys. Fit. 2012, 52, 255-262.

90. Sim, A.Y.; Wallman, K.E.; Fairchild, T.J.; Guelfi, K.J. Effects of high-intensity intermittent exercise training on appetite regulation. Med. Sci. Sports Exerc. 2015, 47, 2441-2449. [CrossRef] [PubMed]

91. Starkoff, B.E.; Eneli, I.U.; Bonny, A.E.; Hoffman, R.P.; Devor, S.T. Estimated aerobic capacity changes in adolescents with obesity following high intensity interval exercise. Int. J. Kinesiol. Sports Sci. 2014, 2, 1-8.

92. Thomas, T.R.; Adeniran, S.B.; Etheridge, G.L. Effects of different running programs on VO2 max, percent fat, and plasma lipids. Can. J. Appl. Sport Sci. 1984, 9, 55-62.

93. Trapp, E.G.; Chisholm, D.J.; Freund, J.; Boutcher, S.H. The effects of high-intensity intermittent exercise training on fat loss and fasting insulin levels of young women. Int. J. Obes. 2008, 32, 684-691. [CrossRef]

94. Wallman, K.; Plant, L.A.; Rakimov, B.; Maiorana, A.J. The effects of two modes of exercise on aerobic fitness and fat mass in an overweight population. Res. Sports Med. 2009, 17, 156-170. [CrossRef]

95. Winding, K.M.; Munch, G.W.; Iepsen, U.W.; Van Hall, G.; Pedersen, B.K.; Mortensen, S.P. The effect on glycaemic control of low-volume high-intensity interval training versus endurance training in individuals with type 2 diabetes. Diabetes Obes. Metab. 2018, 20, 1131-1139. [CrossRef]

96. Zhang, H.; Tong, T.K.; Qiu, W.; Zhang, X.; Zhou, S.; Liu, Y.; He, Y. Comparable Effects of High-intensity interval training and prolonged continuous exercise training on abdominal visceral fat reduction in obese young women. J. Diabetes Res. 2017, 2017, 5071740. [CrossRef]

97. Schoenfeld, B.; Dawes, J. High-intensity interval training: Applications for general fitness training. Strength Cond. J. 2009, 31, 44-46. [CrossRef]

98. Moniz, S.C.; Islam, H.; Hazell, T.J. Mechanistic and methodological perspectives on the impact of intense interval training on post-exercise metabolism. Scand. J. Med. Sci. Sports 2020, 30, 638-651. [CrossRef]

99. Thomas, D.M.; Bouchard, C.; Church, T.; Slentz, C.; Kraus, W.E.; Redman, L.M.; Martin, C.K.; Silva, A.M.; Vossen, M.; Westerterp, K.; et al. Why do individuals not lose more weight from an exercise intervention at a defined dose? An energy balance analysis. Obes. Rev. 2012, 13, 835-847. [CrossRef] [PubMed]

100. King, N.A.; Hopkins, M.; Caudwell, P.; Stubbs, R.J.; Blundell, J.E. Individual variability following 12 weeks of supervised exercise: Identification and characterization of compensation for exercise-induced weight loss. Int. J. Obes. 2008, 32, 177-184. [CrossRef] [PubMed] 
101. Bouchard, C.; Tremblay, A.; Després, J.P.; Thériault, G.; Nadeau, A.; Lupien, P.J.; Moorjani, S.; Prudhomme, D.; Fournier, G. The response to exercise with constant energy intake in identical twins. Obes. Res. 1994, 2, 400-410. [CrossRef]

102. Swift, D.L.; McGee, J.E.; Earnest, C.P.; Carlisle, E.; Nygard, M.; Johannsen, N.M. The effects of exercise and physical activity on weight loss and maintenance. Prog. Cardiovasc. Dis. 2018, 61, 206-213. [CrossRef]

103. Lee, J.J.; Pedley, A.; Hoffmann, U.; Massaro, J.M.; Fox, C.S. Association of changes in abdominal fat quantity and quality with incident cardiovascular disease risk factors. J. Am. Coll. Cardiol. 2016, 68, 1509-1521. [CrossRef]

104. Grgic, J.; Mcllvenna, L.C.; Fyfe, J.J.; Sabol, F.; Bishop, D.J.; Schoenfeld, B.J.; Pedisic, Z. Does aerobic training promote the same skeletal muscle hypertrophy as resistance training? a systematic review and meta-analysis. Sports Med. 2019, 49, 233-254. [CrossRef]

105. Steele, J.; Androulakis-Korakakis, P.; Perrin, C.; Fisher, J.P.; Gentil, P.; Scott, C.; Rosenberger, A. Comparisons of resistance training and "cardio" exercise modalities as countermeasures to microgravity-induced physical deconditioning: New perspectives and lessons learned from terrestrial studies. Front. Physiol. 2019, 10, 1150. [CrossRef]

106. Ekkekakis, P.; Parfitt, G.; Petruzzello, S.J. The pleasure and displeasure people feel when they exercise at different intensities: Decennial update and progress towards a tripartite rationale for exercise intensity prescription. Sports Med. 2011, 41, 641-671. [CrossRef] [PubMed]

107. Rhodes, R.E.; Kates, A. Can the affective response to exercise predict future motives and physical activity behavior? a systematic review of published evidence. Ann. Behav. Med. 2015, 49, 715-731. [CrossRef]

108. Oliveira, B.R.R.; Santos, T.M.; Kilpatrick, M.; Pires, F.O.; Deslandes, A.C. Affective and enjoyment responses in high intensity interval training and continuous training: A systematic review and meta-analysis. PLoS ONE 2018, 13, e0197124. [CrossRef]

109. Nicolaï, S.P.; Kruidenier, L.M.; Leffers, P.; Hardeman, R.; Hidding, A.; Teijink, J.A. Supervised exercise versus non-supervised exercise for reducing weight in obese adults. J. Sports Med. Phys. Fit. 2009, 49, 85-90.

110. Massamba, A.; Dufour, S.P.; Favret, F.; Hureau, T.J. Small-sided games are not as effective as intermittent running to stimulate aerobic metabolism in prepubertal soccer players. Int. J. Sports Physiol. Perform. 2020, 16, 273-279. [CrossRef]

111. Rice, T.; Hong, Y.; Pérusse, L.; Després, J.P.; Gagnon, J.; Leon, A.S.; Skinner, J.S.; Wilmore, J.H.; Bouchard, C.; Rao, D.C. Total body fat and abdominal visceral fat response to exercise training in the HERITAGE Family Study: Evidence for major locus but no multifactorial effects. Metabolism 1999, 48, 1278-1286. [CrossRef]

112. Schumann, M.; Feuerbacher, J.F.; Sünkeler, M.; Freitag, N.; Rønnestad, B.; Doma, K.; Lundberg, T. An updated systematic review and meta-analysis on the compatibility of concurrent aerobic and strength training for skeletal muscle size and function. SportRxiv 2021. [CrossRef] [PubMed]

113. Sabag, A.; Little, J.P.; Johnson, N.A. Low-volume high-intensity interval training for cardiometabolic health. J. Physiol. 2021. [CrossRef]

114. Calbet, J.A.L.; Ponce-González, J.G.; Calle-Herrero, J.; Perez-Suarez, I.; Martin-Rincon, M.; Santana, A.; Morales-Alamo, D.; Holmberg, H.C. Exercise preserves lean mass and performance during severe energy deficit: The role of exercise volume and dietary protein content. Front. Physiol. 2017, 8, 483. [CrossRef]

115. Jakicic, J.M.; Marcus, B.H.; Lang, W.; Janney, C. Effect of exercise on 24-month weight loss maintenance in overweight women. Arch. Intern. Med. 2008, 168, 1550-1559, discussion 1559-1560. [CrossRef] 